\title{
Characteristics of Low-latitude Coronal Holes near the Maximum of Solar Cycle 24
}

Hofmeister, Stefan J.; Veronig, Astrid; Reiss, Martin A.; Temmer, Manuela; Vennerstrøm, Susanne; Vrsnak, Bojan; Heber, Bernd

Published in:

Astrophysical Journal

Link to article, DOI:

$10.3847 / 1538-4357 / 835 / 2 / 268$

Publication date:

2017

Document Version

Publisher's PDF, also known as Version of record

Link back to DTU Orbit

Citation (APA):

Hofmeister, S. J., Veronig, A., Reiss, M. A., Temmer, M., Vennerstrøm, S., Vrsnak, B., \& Heber, B. (2017). Characteristics of Low-latitude Coronal Holes near the Maximum of Solar Cycle 24. Astrophysical Journal, 835(2), [268]. https://doi.org/10.3847/1538-4357/835/2/268

\section{General rights}

Copyright and moral rights for the publications made accessible in the public portal are retained by the authors and/or other copyright owners and it is a condition of accessing publications that users recognise and abide by the legal requirements associated with these rights.

- Users may download and print one copy of any publication from the public portal for the purpose of private study or research.

- You may not further distribute the material or use it for any profit-making activity or commercial gain

- You may freely distribute the URL identifying the publication in the public portal 


\title{
Characteristics of Low-latitude Coronal Holes near the Maximum of Solar Cycle 24
}

\author{
Stefan J. Hofmeister ${ }^{1}$, Astrid Veronig ${ }^{1}$, Martin A. Reiss ${ }^{1}$, Manuela Temmer ${ }^{1}$, Susanne Vennerstrom ${ }^{2}$, \\ Bojan Vršnak ${ }^{3}$, and Bernd Heber ${ }^{4}$ \\ ${ }^{1}$ University of Graz, Institute of Physics, IGAM-Kanzelhöhe Observatory, Graz, Austria; stefan.hofmeister@uni-graz.at \\ ${ }^{2}$ National Space Institute, DTU Space, Denmark \\ ${ }^{3}$ Hvar Observatory, Faculty of Geodesy, Zagreb, Croatia \\ ${ }^{4}$ Universität Kiel, Institut für Experimentelle und Angewandte Physik, Kiel, Germany \\ Received 2016 September 14; revised 2016 December 19; accepted 2016 December 26; published 2017 February 1
}

\begin{abstract}
We investigate the statistics of 288 low-latitude coronal holes extracted from SDO/AIA-193 filtergrams over the time range of 2011 January 01-2013 December 31. We analyze the distribution of characteristic coronal hole properties, such as the areas, mean AIA-193 intensities, and mean magnetic field densities, the local distribution of the SDO/AIA-193 intensity and the magnetic field within the coronal holes, and the distribution of magnetic flux tubes in coronal holes. We find that the mean magnetic field density of all coronal holes under study is $3.0 \pm 1.6 \mathrm{G}$, and the percentaged unbalanced magnetic flux is $49 \pm 16 \%$. The mean magnetic field density, the mean unsigned magnetic field density, and the percentaged unbalanced magnetic flux of coronal holes depend strongly pairwise on each other, with correlation coefficients $c c>0.92$. Furthermore, we find that the unbalanced magnetic flux of the coronal holes is predominantly concentrated in magnetic flux tubes: $38 \%(81 \%)$ of the unbalanced magnetic flux of coronal holes arises from only $1 \%(10 \%)$ of the coronal hole area, clustered in magnetic flux tubes with field strengths $>50 \mathrm{G}(10 \mathrm{G})$. The average magnetic field density and the unbalanced magnetic flux derived from the magnetic flux tubes correlate with the mean magnetic field density and the unbalanced magnetic flux of the overall coronal hole $(c c>0.93)$. These findings give evidence that the overall magnetic characteristics of coronal holes are governed by the characteristics of the magnetic flux tubes.
\end{abstract}

Key words: Sun: corona - Sun: magnetic fields - Sun: UV radiation

\section{Introduction}

Coronal holes are the lowest density regions in the solar atmosphere. Their low density and temperature as compared to the ambient corona results in a reduced emission in the extreme-ultraviolet (EUV) and soft X-ray. Therefore, they appear as dark structures in EUV images and are often identified by intensity-based thresholding techniques (e.g., Krista \& Gallagher 2009; Rotter et al. 2012).

In addition, coronal holes are typically characterized by a large amount of "open" magnetic flux, i.e., magnetic flux, which closes at far distances to the Sun. The open magnetic flux of coronal holes expands rapidly above the bottom of coronal holes due to the low ambient pressure and the low plasma-to-magnetic pressure ratio $\beta$. At a distance between about $1 R_{\odot}$ and $10 R_{\odot}$ above the solar-photosphere surface, plasma is accelerated along the open magnetic field lines to form high-speed solar wind streams, i.e., supersonic plasma flows that transverse our solar system (e.g., review by Kohl et al. 2006). High-speed solar wind streams are the major cause of minor and medium geomagnetic storms at Earth.

The peak velocity of high-speed streams measured at L1 is correlated to the inverse expansion factor of the rapidly expanding magnetic flux tubes of coronal holes evaluated between the lower boundary of coronal holes and the sourcesurface at $2.5 R_{\odot}$ as derived by potential-field source-surface (PFSS) extrapolations (Wang \& Sheeley 1990). In addition, the peak velocity of high-speed streams correlates with the ratio of the mean magnetic field density of the photosphere below a $\mathrm{CH}$ to its flux-tube expansion factor, and with the area of the coronal hole (Nolte et al. 1976; Kojima et al. 2007).

For these reasons, the magnetic field distribution in coronal holes is of high interest. Harvey et al. (1982) and Wang (2009) studied the evolution of the mean magnetic field densities of coronal holes over the solar cycle. They found that the mean magnetic field densities at solar minimum are about $5 \mathrm{G}$, respectively, $0.3-7.2 \mathrm{G}$, and that they are three to five times higher at solar maximum. Bohlin \& Sheeley (1978) studied the magnetic characteristics over the lifetime of coronal holes. They report that the mean magnetic field densities of coronal holes decrease with their age.

Wiegelmann \& Solanki (2004) studied 12 well pronounced coronal holes in comparison to quiet-Sun regions. They report that the relative amount of the total signed magnetic flux to the total unsigned magnetic flux in their coronal holes under study is $77 \pm 14 \%$, i.e., that most of the magnetic flux in coronal holes is open. However, these authors also report that a significant number of closed magnetic loops exists within coronal holes. They have shown that short and low-lying coronal loops are almost as abundant in coronal holes as in quiet-Sun regions, whereas high and long loops are very rare. PFSS extrapolations confirmed that the global magnetic field in coronal holes is predominantly unipolar, open, and expands super-radially with height, whereby the flux-tube expansion factor is highest at the boundary of coronal holes and smallest near the center (e.g., Altschuler et al. 1972; Levine et al. 1977a, 1977b; Wiegelmann \& Solanki 2004). Fainshtein \& Kaigorodov (1994) reported that the flux-tube expansion factor derived at the source-surface, the mean magnetic field density at the base, and the area at the base of the coronal hole depend on each other. Furthermore, they found that the mean magnetic field density derived at the source-surface at $2.5 R_{\odot}$ correlates well with the area at the base of coronal holes.

Correlating the force-free extrapolated magnetic field at different altitudes of coronal holes with Doppler-velocity maps 
derived from $\mathrm{SI}^{+}, \mathrm{C}^{3+}$, and $\mathrm{Ne}^{7+}$ spectral lines, $\mathrm{Tu}$ et al. (2005) found that the solar wind plasma flows out in magnetic funnels at heights between 5 and $20 \mathrm{Mm}$ above the photosphere. Xia et al. (2004) showed that the outflow velocity is statistically higher at higher underlying photospheric magnetic field strengths. Finally, Hassler et al. (1999) showed by spectroscopic observations of the $\mathrm{Ne}^{7+}$ line in a polar coronal hole that high-speed solar wind streams are rooted near the supergranulation boundaries in the chromospheric network lanes below coronal holes.

In this paper, we give a comprehensive statistical overview of 288 records of low-latitude coronal holes detected in EUV images in the time range of 2011 January 01-2013 December 31 , i.e., from the rising phase of solar cycle 24 up to the solar maximum. Note that solar maxima, as inferred from the sunspot numbers, tend to be double-peaked (Gnevyshev 1963, 1977); the first peak of solar cycle 24 appeared in 2012 March corresponding to the northern solar hemisphere and the second, slightly larger peak in 2014 April corresponding to the southern hemisphere. Our data set contains all the coronal holes detected at a cadence of one image per day with areas greater than $10^{10} \mathrm{~km}^{2}$ and center of mass situated within $30^{\circ}$ around the intersection of the solar equator and the central meridian. The coronal holes were allowed to expand beyond the $30^{\circ}$ restriction, but equatorial extensions of polar coronal holes are not included.

We divided this paper into three main parts. The first part presents the distribution of characteristic observational properties of the low-latitude coronal holes, such as their areas, the mean EUV intensity, mean magnetic field densities, the mean unsigned magnetic field densities, the unbalanced magnetic flux, and the percentaged unbalanced magnetic flux, i.e., the relative amount of the total signed to total unsigned magnetic flux. We investigate their correlations, and examine the difference in the statistics between small and large coronal holes. Most notably, we find a strong dependence between the mean magnetic field density, the mean unsigned magnetic field density, and the percentaged unbalance magnetic flux of coronal holes.

In the second part, we investigate the distribution of the AIA-193 intensity and the magnetic field density within coronal holes, in particular, the probability distribution of the AIA-193 intensity along a normalized cross-section of coronal holes, the magnetic polarity of coronal holes at various magnetic levels, and the amount of unbalanced magnetic flux that arises from various magnetic levels. We find that the magnetic field distributions at various magnetic levels of a coronal hole always have the same polarity, and that most of the unbalanced magnetic flux arises from only a small percentage of its area.

In the third part, we present magnetic flux tubes as the origin of the unbalanced magnetic flux of coronal holes. We investigate the distribution of magnetic flux tubes within coronal holes and relate the averaged properties of magnetic flux tubes within coronal holes to the overall magnetic properties of coronal holes. We find strong dependencies between the mean magnetic field density and unbalanced magnetic flux of the flux tubes, of the coronal hole region without the flux tubes, and of the magnetic properties of the overall coronal hole.

The paper is structured as follows. Section 2 describes the data set and the data reduction. Section 3 describes the analysis methods. Section 4 studies the distribution of characteristic observational properties of the low-latitude coronal holes. Section 5 presents the local distribution of the EUV intensity and the magnetic field within the coronal holes. Section 6 studies the distribution and properties of magnetic flux tubes within the coronal holes. Section 7 relates the averaged parameters of magnetic flux tubes to the overall magnetic properties of the coronal holes. Section 8 discusses the results.

\section{Data Sets and Data Reduction}

We use EUV $193 \AA$ filtergrams recorded by the Atmospheric Imaging Assembly (AIA) on board the Solar Dynamics Observatory $(S D O)$ and provided by the Joint Science Operations Center (JSOC), low noise line-of-sight magnetograms recorded by the Helioseismic and Magnetic Imager (HMI) on board SDO and provided by JSOC, and $\mathrm{H} \alpha$ images from the Kanzelhöhe Observatory of Solar and Environmental Research.

To identify coronal holes in EUV images, we use AIA-193 filtergrams. Because of their high contrast between coronal holes and quiet-Sun regions, these filtergrams are often used to identify coronal holes in EUV images by various segmentation techniques (e.g., Krista \& Gallagher 2009; Rotter et al. 2012; Lowder et al. 2014; Verbeeck et al. 2014; Boucheron et al. 2016; Caplan et al. 2016). The AIA-193 $\AA$ filtergrams observe emission from $\mathrm{FeXII}$ ions in the coronal plasma at a temperature of about $1.6 \mathrm{MK}$ (peak response). Images are taken by a $4096 \times 4096$ pixel CCD camera, the spatial resolution is $1.5 \mathrm{arcsec}$ at a plate scale of $0.6 \mathrm{arcsec} / \mathrm{pixel}$ (Lemen et al. 2012).

To study the photospheric magnetic field below coronal holes, we use the HMI-los 720s magnetogram data set. This magnetogram data product is a low noise line-of-sight magnetogram data product derived by the Vector Camera of the HMI instrument. We prefer this data product to the HMI vector magnetograms due to its much higher signal-to-noise ratio at weak magnetic fields. The Vector Camera derives the Stoke's parameters for the full solar disk from the photospheric Fe I line (6173 $\AA$ ). Ten sets of Stoke's parameters recorded at a cadence of $135 \mathrm{~s}$ are then averaged to derive the line-of-sight magnetic field. The plate scale is $0.505 \mathrm{arcsec} / \mathrm{pixel}$ at $4096 \times 4096$ pixels, the spatial resolution is 0.91 arcsec, and the noise level is $5 \mathrm{G} /$ pixel. Note that the noise level primarily arises from photon noise, and therefore decreases with $\sqrt{n}$ by averaging over $n$ pixels (Scherrer et al. 2012; Schou et al. 2012; Couvidat et al. 2016).

To distinguish coronal holes from filaments, we use $\mathrm{H} \alpha$ filtergrams taken by the Kanzelhöhe Observatory. The $\mathrm{H} \alpha$ filtergrams observe emission from neutral hydrogen in the chromosphere at the center of the $\mathrm{H} \alpha$ line $(6563 \AA)$ with an FWHM of $0.7 \AA$. The images are recorded by a $2048 \times 2048$ pixel CCD camera at a plate scale of $1 \mathrm{arcsec} /$ pixel (Pötzi et al. 2015).

All images were normalized to an exposure time of $1 \mathrm{~s}$, rotated to north up, and interpolated to a spatial sampling of $2.4 \mathrm{arcsec} / \mathrm{pixel}$ at $1024 \times 1024$ pixels. The interpolation to $1024 \times 1024$ pixels was performed under the condition of conservation of flux and reduces the noise level of the line-ofsight magnetograms to about $1.25 \mathrm{G}$ per pixel. Due to the different recording times of the AIA-193, HMI-los, and $\mathrm{H} \alpha$ images, the magnetograms and $\mathrm{H} \alpha$ images were co-aligned assuming rigid rotation of the Sun to match the recording time 

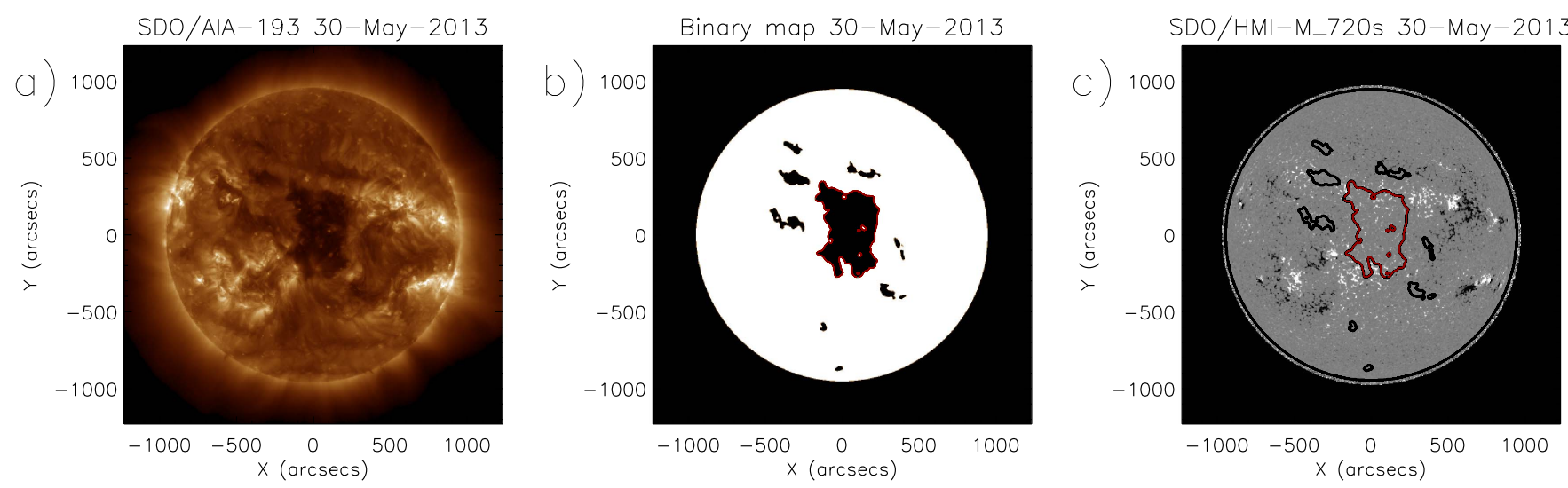

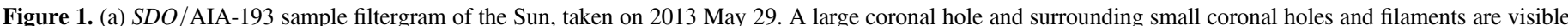

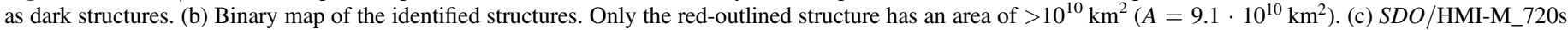
line-of-sight magnetogram of the Sun. The contours of the binary map and the photospheric solar limb are over-plotted.

of the AIA-193 images. The maximum time of de-rotation was 6 minutes for magnetograms and $12 \mathrm{hr}$ for $\mathrm{H} \alpha$ images. Since $\mathrm{H} \alpha$ images are only used for the visual identification of filaments, the error in the de-rotation of $\mathrm{H} \alpha$ images by assuming rigid rotation over $12 \mathrm{hr}$ can be neglected.

\section{Analysis}

\subsection{Extraction of the Coronal Holes}

Two species of structures appear dark in EUV images: coronal holes and filaments. Coronal holes are the lowest density structures in the Sun's corona at comparably low coronal temperatures of about $1 \mathrm{MK}$. Therefore, they appear as dark structures in EUV images, but are not visible in $\mathrm{H} \alpha$ images. In contrast, filaments have a high density, a chromospheric temperature of about $10^{4} \mathrm{~K}$, extending from the Sun's chromosphere to the lower corona. Filaments appear dark in both EUV and $\mathrm{H} \alpha$ images.

To create our data set, we first extracted all dark structures in EUV images for the three-year period under study from 2011 January 01 to 2013 December 31 with a cadence of one image per day. Then we visually classify and exclude filaments within the dark structures by comparison with $\mathrm{H} \alpha$ images. The data set is restricted by the availability of EUV images, $\mathrm{H} \alpha$ images and magnetograms, and covers $98 \%$ of the time range under study.

We extracted the dark structures in the EUV images by an intensity-based thresholding algorithm based on Rotter et al. (2012) and Reiss et al. (2016). The threshold was set to 0.35 times the median of the AIA-193 intensity of the solar disk. The resulting full-disk binary maps of dark structures were post-processed by morphological operators (erosion and dilation), whereby a median function with a kernel of 9 pixels was applied. This morphological operation adds small bright islands within coronal holes to the area of coronal holes and removes very small dark structures, which are most probably neither coronal holes nor filaments, from the full-disk binary map (Rotter et al. 2012). In order to obtain the single structure binary maps, the full-disk binary maps were segmented. Two structures were recognized as individual structures as soon as they are separated by at least one pixel. Finally, the EUV images and magnetograms of the single structures were obtained by cutting the full-disk EUV images and magnetograms according to the single structure binary maps identified in the EUV.

For further analysis, we restricted the single structure data set to structures with an area of more than $1 \times 10^{10} \mathrm{~km}^{2}$ corresponding to a minimum diameter of $100 \mathrm{Mm}$, and with an angular distance of the structure's center of mass to the intersection of the solar equator and central meridian of less than $30^{\circ}$ in order to reduce the effects of projection in the further analysis. Thus, we arrive at a resulting data set of 343 structures. Figure 1 shows an example of the segmentation procedure and resulting coronal holes.

The remaining single structures identified in EUV were then divided into coronal holes and filaments by visual comparison with the $\mathrm{H} \alpha$ images. Since filament channels are sometimes visible in EUV before the corresponding channel is visible in $\mathrm{H} \alpha$, i.e., before the channel is filled with dense cool plasma, we inspected the $\mathrm{H} \alpha$ images within a time range of \pm 2 days. If the full structure was visible in the $\mathrm{H} \alpha$ images within a time range of \pm 2 days, the structure was labelled as a filament. If only part of the structure was visible in $\mathrm{H} \alpha$, the structure was excluded from further analysis. If the structure was not visible in $\mathrm{H} \alpha$, but had an elongated shape and a balanced magnetic flux, like we expect from filaments, the structure was also excluded from further analysis. All structures remaining were labelled as coronal holes.

In total, our data set includes 288 records of dark structures that were identified as coronal holes, 49 dark structures, which were identified as filaments, and 6 dark structures, which could not be uniquely assigned, were excluded from further analysis.

\subsection{Analysis of the Characteristic Properties of the Coronal Holes}

We analyzed the distribution of characteristic properties in the coronal hole data set, i.e., the areas, latitudes of the center of mass, the mean EUV intensities, the mean value and skewness of the distribution of the radial magnetic field in the photosphere, and the magnetic flux.

We denote a pixel within a coronal hole as $i$, its projected area as $A_{i, \text { proj, }}$, its AIA-193 intensity as $I_{193, i}$, its heliographic latitude as $\varphi_{i}$, its line-of-sight magnetic field density as $B_{i, \text { los }}$, and the total number of pixels within the coronal hole as $n$.

First, in order to avoid systematic errors due to the different latitudes of the coronal holes, we presume the magnetic field to 
be radial and correct each pixel for projection effects:

$$
\begin{aligned}
A_{i} & =\frac{A_{i, \mathrm{proj}}}{\cos \alpha_{i}}, \\
B_{i} & =\frac{B_{i, \mathrm{los}}}{\cos \alpha_{i}},
\end{aligned}
$$

where $\alpha_{i}$ is the heliographic angular distance of the pixel to the center of the solar disk as seen from $S D O$.

Then, for each coronal hole, we calculated the projectioncorrected area $A$, the latitude of its center of mass $\varphi$, and the mean EUV intensity in the $193 \AA$ filtergrams $\bar{I}_{193}$.

Furthermore, we calculated the projection-corrected unbalanced magnetic flux $\Phi$, the total unsigned magnetic flux $\Phi_{\mathrm{t}}$, and the percentaged unbalanced magnetic flux $\Phi_{\mathrm{pu}}$, by

$$
\begin{gathered}
\Phi=\sum_{i} B_{i} A_{i}, \\
\Phi_{\mathrm{t}}=\sum_{i}\left|B_{i} A_{i}\right|, \\
\Phi_{\mathrm{pu}}=\frac{\Phi}{\Phi_{\mathrm{t}}} .
\end{gathered}
$$

The unbalanced magnetic flux gives the magnetic flux, which is not closed within the coronal hole. Under the standard assumption that only magnetic flux near the border of the coronal hole closes with neighboring regions, the unbalanced magnetic flux is a reasonable estimate of the open magnetic flux. The percentaged unbalanced magnetic flux gives the percentage of magnetic flux, which is not closed within the coronal hole.

Finally, we calculated the projection-corrected mean magnetic field density $\bar{B}$ and the projection-corrected mean unsigned magnetic field density $\bar{B}_{\text {us }}$ in the photosphere below the coronal hole,

$$
\begin{gathered}
\bar{B}=\frac{\Phi}{A}, \\
\bar{B}_{\mathrm{us}}=\frac{\Phi_{\mathrm{t}}}{A},
\end{gathered}
$$

and the skewness $\operatorname{Skew}(B)$ of the magnetic field distribution. In our context, the skewness is a measure of the distinctness of a dominant polarity.

\subsection{Analysis of the Pixel Distribution within the Coronal Holes}

Whereas in Section 3.2 we analyze the distribution of characteristic properties of coronal holes, here we analyze the distribution of AIA-193 and magnetogram pixels within the coronal holes. To do so, we calculate for each coronal hole a normalized histogram. Then, we stack the normalized histograms of all coronal holes, whereby all histograms are weighted equally, i.e., small coronal holes get the same weight as large coronal holes. The projection of the stacked histograms gives us a median representation of the histograms and a $1 \sigma$ and a $2 \sigma$ range in which the histograms lie, i.e., a probability distribution of the histograms. This approach provides us with a characterization of the average pixel distribution within all coronal holes under study.

First, we analyzed the distribution of the EUV intensity within coronal holes. For each coronal hole, we created a histogram of the pixels of the AIA-193 intensity $I_{193, i}$, and normalized the histograms by their total number of pixels to obtain for each coronal hole the percentage of pixels belonging to each intensity bin. Then we calculated separately from all coronal holes the median value and the $1 \sigma$ and $2 \sigma$ ranges of the percentages of pixels for each intensity bin.

Next, we analyzed the EUV intensities along the major axis of coronal holes. First, we calculated the direction of the major axis by using a least-square fit on the longitude-latitude tupels of all pixels of the coronal hole. We calculated the length of all cross-sections parallel to the derived direction to get the longest cross-section, which we denote as the major axis. We define the width of the coronal hole as the number of pixels along the longest cut perpendicular to the major axis. The AIA-193 intensity along the major axis was then derived by averaging over $\pm 1 \%$ of the width of the coronal hole perpendicular to major axis. For each coronal hole, we derived the AIA-193 intensity versus the position along the normalized major axis. We then calculated from all coronal holes the representative median AIA-193 intensity and the $1 \sigma$ and $2 \sigma$ ranges for the normalized positions along the major axis.

Furthermore, we analyzed the distribution of the magnetic field densities within coronal holes. Since the dominant polarity of coronal holes can be positive or negative, we transform the magnetic field densities $B_{i}$ within a coronal hole so that positive values always belong to the dominant polarity of the coronal hole:

$$
B_{+}=\left\{\begin{array}{ll}
B_{i} & \text { for } \bar{B} \geqslant 0 \\
-B_{i} & \text { for } \bar{B}<0
\end{array} .\right.
$$

For each coronal hole, we created a histogram of $B_{+}$.

Next, we analyzed the distinctiveness of the dominant polarity at various magnetic field density levels within coronal holes. We define the magnetic flux arising from a given signed magnetic field density level $l$ as

$$
\Phi_{\mathrm{lv}}(l)=\sum_{\left|-B_{+}+l\right|<1 \mathrm{G}} B_{+} A_{i},
$$

where $\left|-B_{+}+l\right|<1 \mathrm{G}$ corresponds to the binning of the magnetic field density values $B_{+}$. Then, we denote the fraction of the magnetic flux arising from a given magnetic field density level $l$, which has the same polarity as the overall coronal hole as the polarity dominance $\Phi_{\mathrm{pd}}(l)$ of the magnetic field density level $l$,

$$
\Phi_{\mathrm{pd}}(l)=\frac{\left|\Phi_{\mathrm{lv}}(l)\right|}{\left|\Phi_{\mathrm{lv}}(l)\right|+\left|\Phi_{\mathrm{lv}}(-l)\right|} .
$$

A polarity dominance of $\Phi_{\mathrm{pd}}(l)=0.5$ means that the magnetic flux arising from the magnetic field density level $l$ is balanced, whereas a polarity dominance of $\Phi_{\mathrm{pd}}(l)=1 \quad\left(\Phi_{\mathrm{pd}}(l)=0\right)$ corresponds to a unipolar magnetic flux arising from the magnetic field density level $l$ with the same (opposite) polarity as the overall coronal hole.

Finally, we analyzed the fraction of the unbalanced magnetic flux $\Phi$ of a coronal hole that arises from all pixels above a given threshold magnetic field density $b, \Phi_{\mathrm{fu}}(b)$ :

$$
\Phi_{\mathrm{fu}}(b)=\frac{\sum_{\left|B_{+}\right| \geqslant b} B_{+} A_{i}}{\Phi} .
$$




\subsection{Analysis of the Distribution of Magnetic Flux Tubes within the Coronal Holes}

We investigated the characteristics of magnetic flux tubes within each coronal hole. We extracted the magnetic flux tubes by a thresholding technique on the magnetograms of the coronal holes at different global thresholds of thr $=i \cdot 10 \mathrm{G}$, $i \in\{1,2, \ldots, 8\}$. First, all pixels in the magnetogram with $\left|B_{i}\right|<$ thr were set to Not a Number. Each island remaining within the magnetograms is defined as a magnetic flux tube. The resulting magnetogram was segmented, whereby two flux tubes were identified as individual flux tubes as soon as they were separated by at least one pixel. Note that the flux tubes extracted and their parameters derived depend on the magnetic extraction threshold, thr.

For each flux tube extracted at a threshold thr, we calculated the following parameters: its projection-corrected area $A_{\circ, \text { thr }}$, its projection-corrected mean magnetic field density $\bar{B}_{\circ, \text { thr }}$, and its projection-corrected magnetic flux $\Phi_{\mathrm{o}, \mathrm{thr}}$ (see Section 3.2).

For each coronal hole, we counted the number of flux tubes $\tilde{N}_{\text {o,thr. }}$. We calculated the total area $\tilde{A}_{\circ \text {,thr }}$ of the coronal hole that is covered by flux tubes, their summed unbalanced magnetic flux $\tilde{\Phi}_{\circ, \text { thr }}$, their summed total unsigned magnetic flux $\tilde{\Phi}_{\circ, t, t h r}$, and their averaged magnetic field density $\tilde{B}_{\circ, \text { thr }}$, by summing (averaging) over all pixels that belong to magnetic flux tubes extracted at a given threshold thr:

$$
\begin{gathered}
\tilde{A}_{\circ, \mathrm{thr}}=\sum_{\left|B_{i}\right| \geqslant \mathrm{thr}} A_{i} \\
\tilde{\Phi}_{\circ, \mathrm{thr}}=\sum_{\left|B_{i}\right| \geqslant \mathrm{thr}} B_{i} A_{i} \\
\tilde{\boldsymbol{\Phi}}_{\circ, t \mathrm{thr}}=\sum_{\left|B_{i}\right| \geqslant \mathrm{thr}}\left|\boldsymbol{B}_{i} \boldsymbol{A}_{i}\right|, \\
\tilde{B}_{\circ, \mathrm{thr}}=\frac{1}{\tilde{A}_{\circ, \mathrm{thr}}} \sum_{\left|B_{i}\right| \geqslant \mathrm{thr}} B_{i} A_{i} .
\end{gathered}
$$

In addition, we calculated the mean magnetic field density of the coronal hole without the flux tubes, which we denote as "quiet" coronal hole regions:

$$
\bar{B}_{\mathrm{qu}, \mathrm{thr}}=\frac{1}{\left(A-\tilde{A}_{\circ, \mathrm{thr}}\right)} \sum_{\left|B_{i}\right|<\mathrm{thr}} B_{i} A_{i},
$$

where $A$ is the area of the coronal hole.

Finally, for each coronal hole, we estimated the fraction of the magnetic flux arising from all flux tubes, which is unbalanced, i.e., the percentaged unbalanced magnetic flux of the flux tubes

$$
\tilde{\Phi}_{\mathrm{pu}, \mathrm{thr}}=\frac{\tilde{\Phi}_{\mathrm{o}, \mathrm{thr}}}{\tilde{\Phi}_{\mathrm{o}, t, \mathrm{thr}}} .
$$

Note that under the condition of similar absolute unbalanced magnetic fluxes $\left|\Phi_{\circ, \text { thr }}\right|$ of the flux tubes, $\tilde{\Phi}_{\mathrm{pu}, \text { thr }}$ is also an estimate of the number of flux tubes that are open and on the number of flux tubes that are closed loops.

An overview of all properties defined is given in Table 1.

\section{Characteristic Properties of Low-latitude Coronal Holes}

In this section, we present the statistics of characteristic properties of the 288 records of low-latitude coronal holes from 2011 January 01 to 2013 December 31. In particular, we present the area, the latitude, the mean AIA-193 intensity, the
Table 1

Overview of the Parameters Defined in Sections 3.2-3.4

\begin{tabular}{lll}
\hline \hline Properties of coronal holes: & \\
$\mathrm{A}$ & $=\sum_{i} A_{i}$ & Area \\
$\varphi$ & $=\frac{1}{A} \sum_{i} \varphi_{i} A_{i}$ & Latitude of center of mass \\
$I_{193}$ & $=\frac{1}{n} \sum_{i} I_{193, i}$ & Mean AIA-193 intensity \\
$\Phi$ & $=\sum_{i} A_{i} B_{i}$ & Unbalanced magnetic flux \\
$\Phi_{\mathrm{pu}}$ & $=\frac{\sum_{i} A_{i} B_{i}}{\sum_{i}\left|A_{i} B_{i}\right|}$ & percentaged unbalanced magnetic flux \\
$\bar{B}$ & $=\frac{\Phi}{A}$ & Mean magnetic field density \\
$\bar{B}_{\mathrm{us}}$ & $\frac{\sum_{i}\left|A_{i} B_{i}\right|}{A}$ & Mean unsigned magnetic field density
\end{tabular}

Pixel distribution within coronal holes:

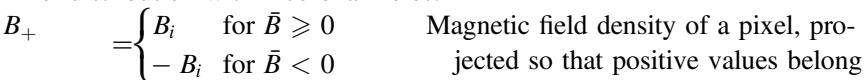
to the dominant polarity of the coronal hole

$\Phi_{\mathrm{pd}}(l) \quad=\frac{\sum_{l-1 \mathrm{G}<B_{+}<l+1 \mathrm{G}} B_{+} A_{i}}{\sum_{l-1 \mathrm{G}<\left|B_{+}\right|<l+1 \mathrm{G}\left|B_{+} A_{i}\right|}}$

$\Phi_{\mathrm{fu}}(b) \quad=\frac{1}{\Phi} \sum_{\left|B_{+}\right| \geqslant b} B_{+} A_{i}$

\begin{tabular}{|c|c|c|}
\hline$A_{\circ, \mathrm{thr}}$ & $=\sum_{f} A_{i}$ & Area of a single flux tube \\
\hline$\Phi_{\circ, \mathrm{thr}}$ & $=\sum_{f} B_{i} A_{i}$ & Magnetic flux of a single flux tube \\
\hline $\bar{B}_{\circ, \mathrm{thr}}$ & $=\frac{\Phi_{\circ, \mathrm{thr}}}{A_{\circ, \mathrm{thr}}}$ & $\begin{array}{l}\text { Mean magnetic field density of a single } \\
\text { flux tube }\end{array}$ \\
\hline$\tilde{N}_{\circ, \text { thr }}$ & & Number of flux tubes of a coronal hole \\
\hline$\tilde{A}_{\circ, \text { thr }}$ & $=\sum_{\left|B_{i}\right| \geqslant \mathrm{thr}} A_{i}$ & $\begin{array}{l}\text { Total area covered by all flux tubes of a } \\
\text { coronal hole }\end{array}$ \\
\hline$\tilde{\Phi}_{\circ, \text { thr }}$ & $=\sum_{\left|B_{i}\right| \geqslant \operatorname{thr}} B_{i} A_{i}$ & $\begin{array}{l}\text { Unbalanced magnetic flux derived from } \\
\text { all flux tubes of a coronal hole }\end{array}$ \\
\hline$\tilde{\Phi}_{\mathrm{pu}, \mathrm{thr}}$ & $=\frac{\sum_{\left|B_{i}\right| \geqslant \operatorname{thr}} B_{i} A_{i}}{\sum_{\left|B_{i}\right| \geqslant \operatorname{thr}}\left|B_{i} A_{i}\right|}$ & $\begin{array}{l}\text { Percentaged unbalanced magnetic flux } \\
\text { derived from all flux tubes of a cor- } \\
\text { onal hole }\end{array}$ \\
\hline$\tilde{B}_{\circ, \mathrm{thr}}$ & $=\frac{1}{\tilde{A}_{\circ}, \mathrm{thr}} \sum_{\left|B_{i}\right| \geqslant \mathrm{thr}} B_{i} A_{i}$ & $\begin{array}{l}\text { Average magnetic field density derived } \\
\text { from all flux tubes of a coronal hole }\end{array}$ \\
\hline $\bar{B}_{\mathrm{qu}, t h r}$ & $=\frac{1}{A-\tilde{A}_{\circ, \mathrm{thr}}} \sum_{\left|B_{i}\right|<t h r} B_{i} A_{i}$ & $\begin{array}{l}\text { Mean magnetic field density of the cor- } \\
\text { onal hole region without the flux } \\
\text { tubes }\end{array}$ \\
\hline
\end{tabular}

Polarity dominance of the pixels at the given magnetic field density level $l$

Fraction of the unbalanced magnetic flux that arises from pixels with $B_{+} \geqslant b$

Magnetic flux tubes extracted at a threshold, thr:

Note. The subscript $i$ denotes a pixel of a coronal hole, $f$ a pixel of a flux tube, $A_{i}$ its projection-corrected area, $B_{i}$ its projection-corrected mean magnetic field density, $I_{193, i}$ its mean AIA-193 intensity, $\varphi_{i}$ its latitude, and $\mathrm{n}$ the total number of pixels within the coronal hole.

mean magnetic field density, the mean unsigned magnetic field density, the skewness of magnetic field distribution, the unbalanced magnetic flux, and the percentaged unbalanced magnetic flux. In addition, we investigate the correlations between characteristic properties, and the differences in the distributions between small and large coronal holes.

\subsection{Areas, Latitudes, and Polarities}

Figure 2 gives an overview of the data set of the low-latitude coronal holes, i.e., the distribution of the areas, latitudes, and polarities of all coronal holes under study.

Figure 2(a) shows the distribution of the deprojected areas $A$ of the coronal holes. The median area is $2.39 \cdot 10^{10} \mathrm{~km}^{2}$. Only $18 \%$ of all coronal holes under study had areas of $A>5 \cdot 10^{10} \mathrm{~km}^{2}$. The largest coronal hole detected had an area of $1.4 \cdot 10^{11} \mathrm{~km}^{2}$, i.e., covering $2.25 \%$ of the visible solar hemisphere.

Figure 2(b) shows the heliographic latitudes $\varphi$ of the center of mass of coronal holes versus their areas $A$. In the time range 

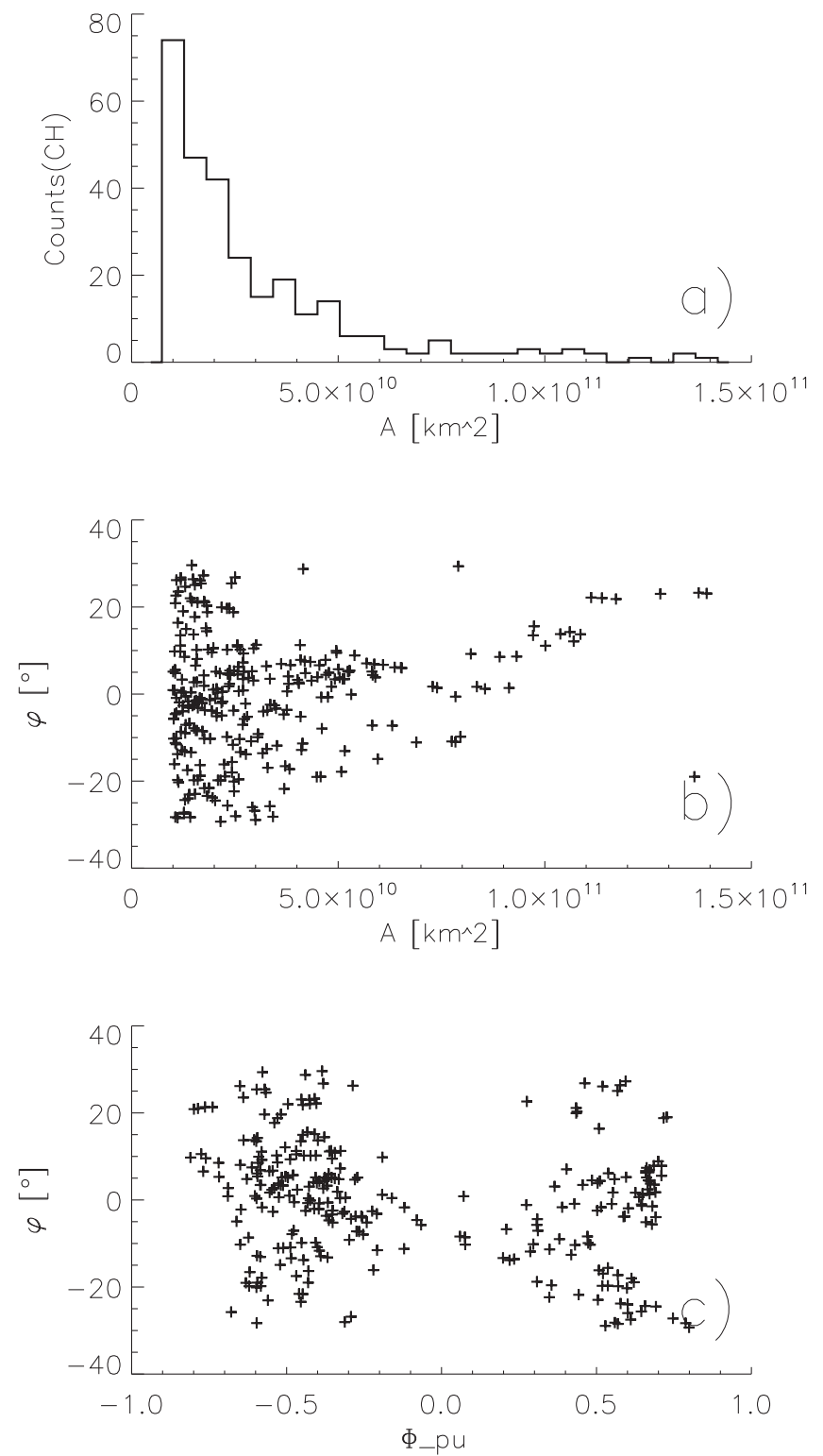

Figure 2. Distribution of the areas of the coronal holes (a). Scatter plots of the heliographic latitude of the center of mass of coronal holes vs. their areas (b) and vs. their percentaged unbalanced magnetic flux (c).

under study, large coronal holes appeared predominantly on the northern hemisphere, whereas small and medium sized coronal holes appeared at both hemispheres.

Figure 2(c) shows the latitudes $\varphi$ of the center of mass of coronal holes versus their percentaged unbalanced magnetic flux $\Phi_{\mathrm{pu}}$, which corresponds to their magnetic polarity. In the time range under study, i.e., the rising phase of solar cycle 24 up to solar maximum, coronal holes appeared with both polarities in each of the two hemispheres at almost all times. This can be explained by the reversal of the magnetic polarity of polar coronal holes during the time range under study. Coronal holes with a small percentaged unbalanced magnetic flux, i.e., a weakly pronounced polarity, only appeared near the solar equator, with a medium percentaged unbalanced magnetic flux at all latitudes, and with a high percentaged unbalanced magnetic flux only at some distance to the solar equator. Note that this plot is not symmetric to the solar equator, but to $\approx 5^{\circ}$ south.

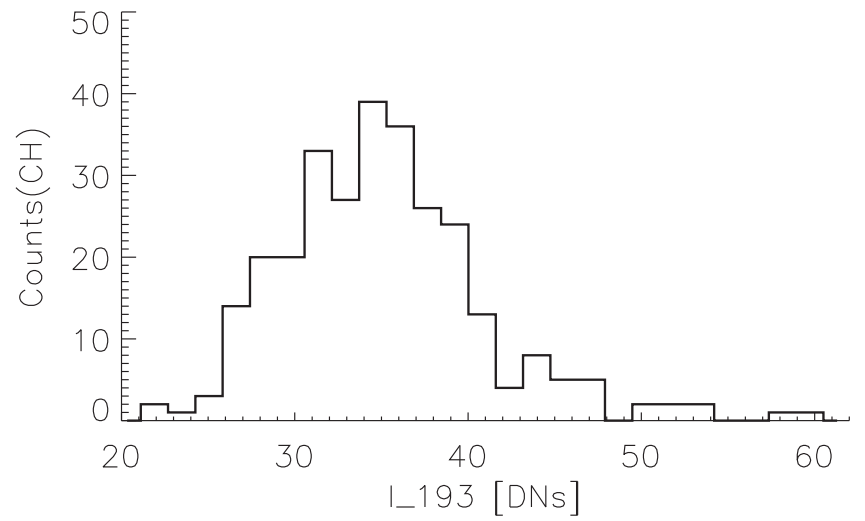

Figure 3. Distribution of the mean AIA-193 intensities derived from all coronal holes.

\subsection{Mean AIA-193 Intensity}

Figure 3 shows the distribution of the mean AIA-193 intensities $\bar{I}_{193}$ of all coronal holes under study. The mean AIA193 intensity is $36 \pm 6$ DNs. Only $3 \%$ of all coronal holes had mean intensities of $\bar{I}_{193}>50 \mathrm{DNs}$; the brightest coronal hole had a mean intensity of $60 \mathrm{DNs}$. For comparison, the most common pixel intensity of the solar disk in all the AIA-193 $\AA$ filtergrams in our data set, i.e., the quiet-Sun intensity, is in the range of $109 \pm 16 \mathrm{DNs}$, and the median Sun intensity is $150 \pm 17$ DNs.

\subsection{Magnetic Properties}

Figures 4(a) and (b) show the distribution of the unbalanced magnetic flux $\Phi$ and the percentaged unbalanced magnetic flux $\Phi_{\mathrm{pu}}$ of coronal holes. The absolute unbalanced magnetic flux $|\Phi|$ ranges from $2.2 \cdot 10^{19}$ to $4.7 \cdot 10^{21} \mathrm{Mx}$ with a mean value of $9.9 \pm 9.4 \cdot 10^{20} \mathrm{Mx}$. The percentaged unbalanced magnetic flux $\Phi_{\text {ro }}$ shows two broad peaks at $-0.46 \pm 0.15$ and $0.52 \pm 0.17$, implying that, on average, $\approx 49 \%$ of the magnetic flux piercing through the photosphere is not closed within the coronal hole. $3 \%$ of all coronal holes had absolute values of the percentaged unbalanced magnetic flux $\left|\Phi_{\mathrm{pu}}\right|>0.75$, and $2 \%$ of all coronal holes had $\left|\Phi_{\mathrm{pu}}\right|<0.10$.

Figures 4(c) and (d) show the distribution of the mean magnetic field densities $\bar{B}$, and the skewness $\operatorname{skew}(B)$ of the magnetic field distributions of coronal holes. The mean value of $|\bar{B}|$ is $2.97 \pm 1.55 \mathrm{G}$. The mean value of $|\operatorname{skew}(B)|$ is $6.6 \pm 1.6$, indicating that, in general, coronal holes have a distinct dominant polarity.

\subsection{Correlation of Characteristic Properties}

Figures 5(a), (b), and (c) show the mean magnetic field densities $\bar{B}$ of the coronal holes versus their areas $A$, the mean AIA-193 intensities $\bar{I}_{193}$ versus the areas $A$, and the mean AIA193 intensities $\bar{I}_{193}$ versus the mean magnetic field densities $\bar{B}$. These three parameters do not correlate. However, we note that there is some difference between small and large coronal holes, which is discussed in Section 4.5.

Figure 5(d) shows the unbalanced magnetic flux $\Phi$ of coronal holes versus their areas $A$. The absolute values of the unbalanced magnetic flux correlate with the areas with a Pearson correlation coefficient of 0.87 , the dependency can be 

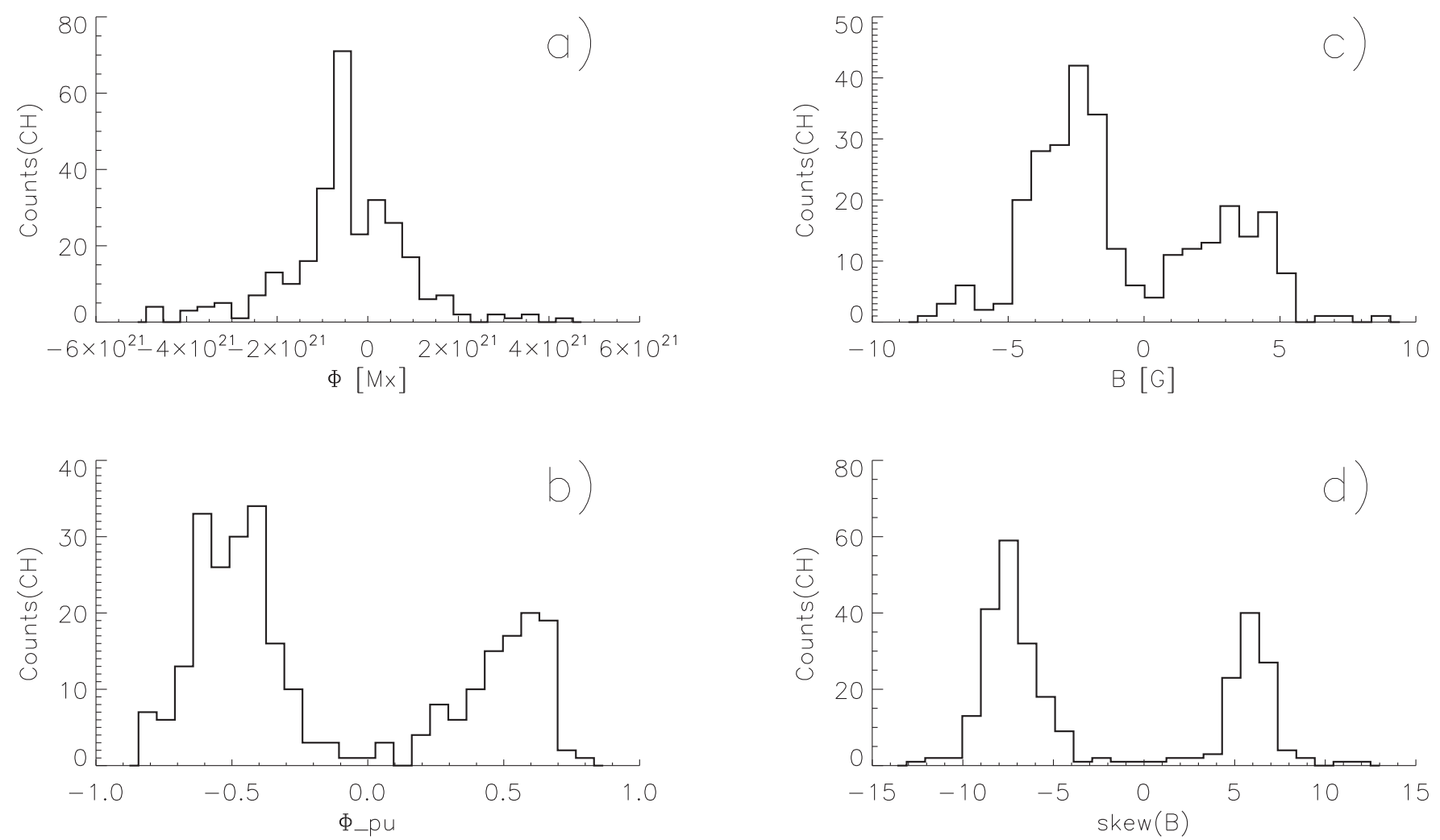

Figure 4. Distribution of the unbalanced magnetic flux (a), percentaged unbalanced magnetic flux (b), and mean magnetic field densities (c), and skewness of the magnetic field distribution (d), derived from all coronal holes.

expressed as

$$
|\Phi|[\mathrm{Mx}]=-4.26 \cdot 10^{19}+3.45 \cdot 10^{10} \cdot A\left[\mathrm{~km}^{2}\right]
$$

with a root-mean-square error (RMSE) of $4.79 \cdot 10^{20} \mathrm{Mx}$. However, $66 \%$ of the coronal holes are rather small and have an absolute value of unbalanced magnetic flux of less than $1 \cdot 10^{21} \mathrm{Mx}$. Therefore, this relationship is not suited to predict the unbalanced magnetic flux of a coronal hole, but only to estimate the magnitude of the unbalanced magnetic flux.

Figure 6(a) shows the mean unsigned magnetic flux $B_{\text {us }}$ of coronal holes versus the absolute values of their mean magnetic field densities. A distinct, linear correlation is apparent, with a Pearson's correlation coefficient of 0.976 . The relationship can be expressed as

$$
B_{\mathrm{us}}[\mathrm{G}]=3.17+0.86|\bar{B}|
$$

with an RMSE of $0.30 \mathrm{G}$.

Figures 6(b) and (c) show the percentaged unbalanced magnetic flux of coronal holes versus the absolute values of the mean magnetic field densities and versus the mean unsigned magnetic field densities. Distinct relationships are also apparent here, with Spearman's rank correlation coefficients of 0.983 and 0.920 . The relationships can be expressed as

$$
\begin{aligned}
\left|\Phi_{\mathrm{pu}}\right| & =\frac{|\bar{B}|[\mathrm{G}]}{3.17+0.86|\bar{B}|[\mathrm{G}]}, \\
\left|\Phi_{\mathrm{pu}}\right| & =\frac{-3.69+1.17 \bar{B}_{\mathrm{us}}[\mathrm{G}]}{\bar{B}_{\mathrm{us}}[\mathrm{G}]},
\end{aligned}
$$

with RMSEs of 0.025 , respectively, 0.064. Since the percentaged unbalanced magnetic flux $\Phi_{\text {pu }}$ can be approximated by the ratio of the mean magnetic field density to the mean unsigned magnetic field density $\bar{B} / \bar{B}_{\text {us }}$, Equations (20) and (21) are directly related to Equation (19). Note that Equation (19) implies that the mean magnetic field density of a coronal hole strongly depends on its mean unsigned magnetic field density, and Equation (20) implies that the amount of unbalanced magnetic flux relative to the total unsigned flux inside a coronal hole can be predicted purely by the mean magnetic field density!

\subsection{Differences in the Characteristic Properties of Small and Large Coronal Holes}

Figures 5(a), (b), and (d) show differences in the distribution of characteristic properties of small coronal holes, which we define as the 115 coronal holes with areas $A<2 \cdot 10^{10} \mathrm{~km}^{2}$, and the 12 large coronal holes with $A>1 \cdot 10^{11} \mathrm{~km}^{2}$.

Small coronal holes have a wide range of mean AIA-193 intensities $\bar{I}_{193}$ ranging from 24 to 60 DNs (Figure 5(b)). The mean magnetic field densities $|\bar{B}|$ range from 0.2 to $8.7 \mathrm{G}$ (Figure 5(a)). The absolute values of the percentaged unbalanced magnetic flux $\left|\Phi_{\text {pu }}\right|$ takes values of $0.06-0.81$, i.e., the magnetic flux piercing through the photosphere can be balanced well and can also be almost unipolar. The absolute unbalanced magnetic flux $|\Phi|$ is comparably small, ranging from $2.2 \cdot 10^{19}$ to $1.3 \cdot 10^{21} \mathrm{Mx}$ (Figure $5(\mathrm{~d})$ ). Note that we cannot state if the small coronal holes with an almost balanced magnetic flux are coronal holes at their birth/end or if they are artefacts.

In contrast, large coronal holes always appear as dark structures with $\bar{I}_{193}<37$ DNs (Figure 5(b)). They have neither very small nor very large magnetic field densities, $|\bar{B}|$ ranges from 2.3 to $4.3 \mathrm{G}$ (Figure 5(a)). The absolute values of the 

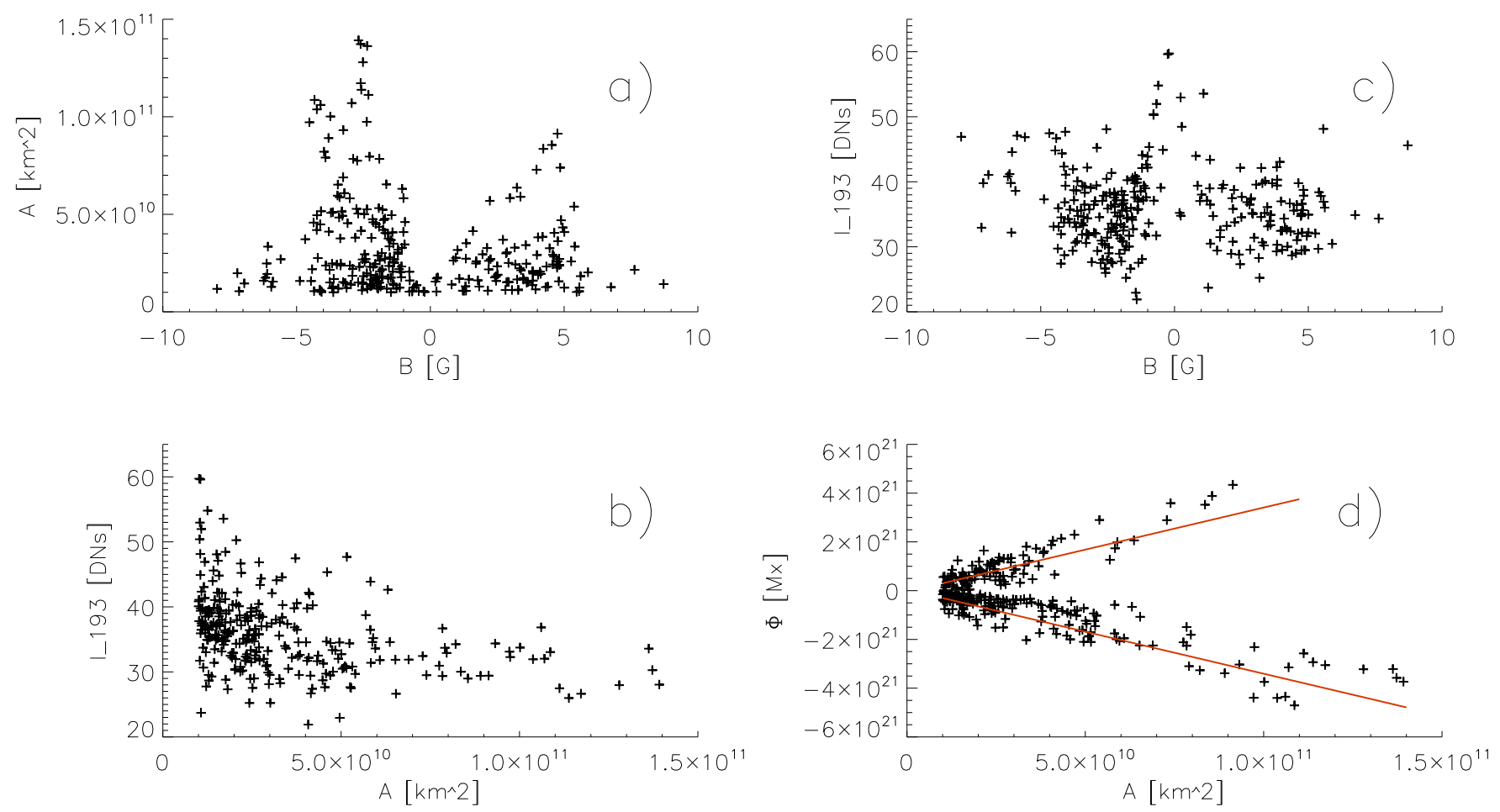

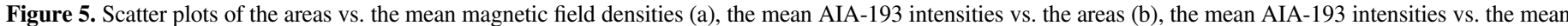

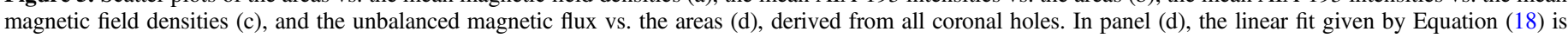
over-plotted in red.

percentaged unbalanced magnetic flux $\left|\Phi_{\text {pu }}\right|$ ranges from 0.40 to 0.64 , and they always have an absolute unbalanced magnetic flux of $|\Phi|>2.5 \cdot 10^{21} \mathrm{Mx}$. This findings show that the characteristics of large coronal holes are much more constrained than the characteristics of small coronal holes.

\section{Pixel Distribution Inside the Low-latitude Coronal Holes}

In this section, we present the distribution of pixels inside coronal holes. First, we derive the distribution of AIA-193 and magnetogram pixels for each coronal hole separately, and then stack the normalized distributions of all coronal holes with equal weight to obtain the median representation and the variance of the distribution of pixels. Note that this kind of representation represents the probability distributions. In particular, we present the distribution of the AIA-193 $\AA$ intensity inside coronal holes, the distribution of AIA-193 $\AA$ intensity along the major axis of coronal holes, the distribution of the magnetic field density inside coronal holes, the distinctiveness of the dominant polarity at various magnetic levels, and the percentage of the unbalanced magnetic flux that arises from various magnetic levels.

\subsection{AIA-193 Intensity}

Figure 7(a) shows the stacked AIA-193 intensity histograms of coronal holes. The solid line gives the median AIA-193 intensity histogram, the dashed line gives the $1 \sigma$ range, i.e., the range that contains $68 \%$ of all AIA-193 intensity histograms. The AIA-193 intensity is distributed broadly inside coronal holes, ranging from about 15 to $70 \mathrm{DNs}$; the mode is at $27 \mathrm{DNs}$. $1 \%$ of all pixels have intensities of $I_{193, i}<16$ DNs, $10 \%$ have intensities of $I_{193, i}<20 \mathrm{DNs}, 10 \%$ have intensities of $I_{193, i}>50 \mathrm{DNs}$, and $1 \%$ have intensities of $I_{193, i}>65 \mathrm{DNs}$.

\subsection{AIA-193 Intensity Along the Major Axis}

Figure 7(b) shows the stacked AIA-193 intensity distributions along the major axis of the coronal holes. For comparison, the horizontal stripes give the distribution of the median AIA193 intensities of the entire solar disks ( $150 \pm 17 \mathrm{DNs})$, which is also an estimate of the median AIA-193 intensities of quietSun regions. At the border of coronal holes, the median representation of the intensities steeply decreases from the quiet-Sun intensity to a low intensity of about 40 DNs. After the steep decrease, the median AIA-193 intensity stays flat, i.e., coronal holes generally do not reveal an intensity minimum at their center. We further investigated the AIA-193 intensity distribution by inspecting the AIA-193 intensity distribution along the cross-section for each coronal hole separately. We found a wide variety on intensity distributions: from almost homogeneous intensity distributions without distinct intensity minimum, intensity distributions with large fluctuations, which most probably arise from coronal bright points located inside of coronal holes, intensity distributions with one and more distinct intensity minima at different locations at the cross-section, intensity distributions that rise sharply at the border of coronal holes, to intensity distributions that rise slowly and steadily at the borders. Thus, Figure 7(b) represents only the statistical cross-section of the AIA-193 intensity as derived by the superposed epoch analysis, but does not hold as a prototype cross-section of coronal holes.

\subsection{Magnetic Field Density}

Figure 8(a) shows the stacked histograms of the magnetic field density of coronal holes. Positive values of $B_{+}$represent pixels with the same polarity as the dominant polarity of the coronal hole, negative values of $B_{+}$pixels with the opposite 

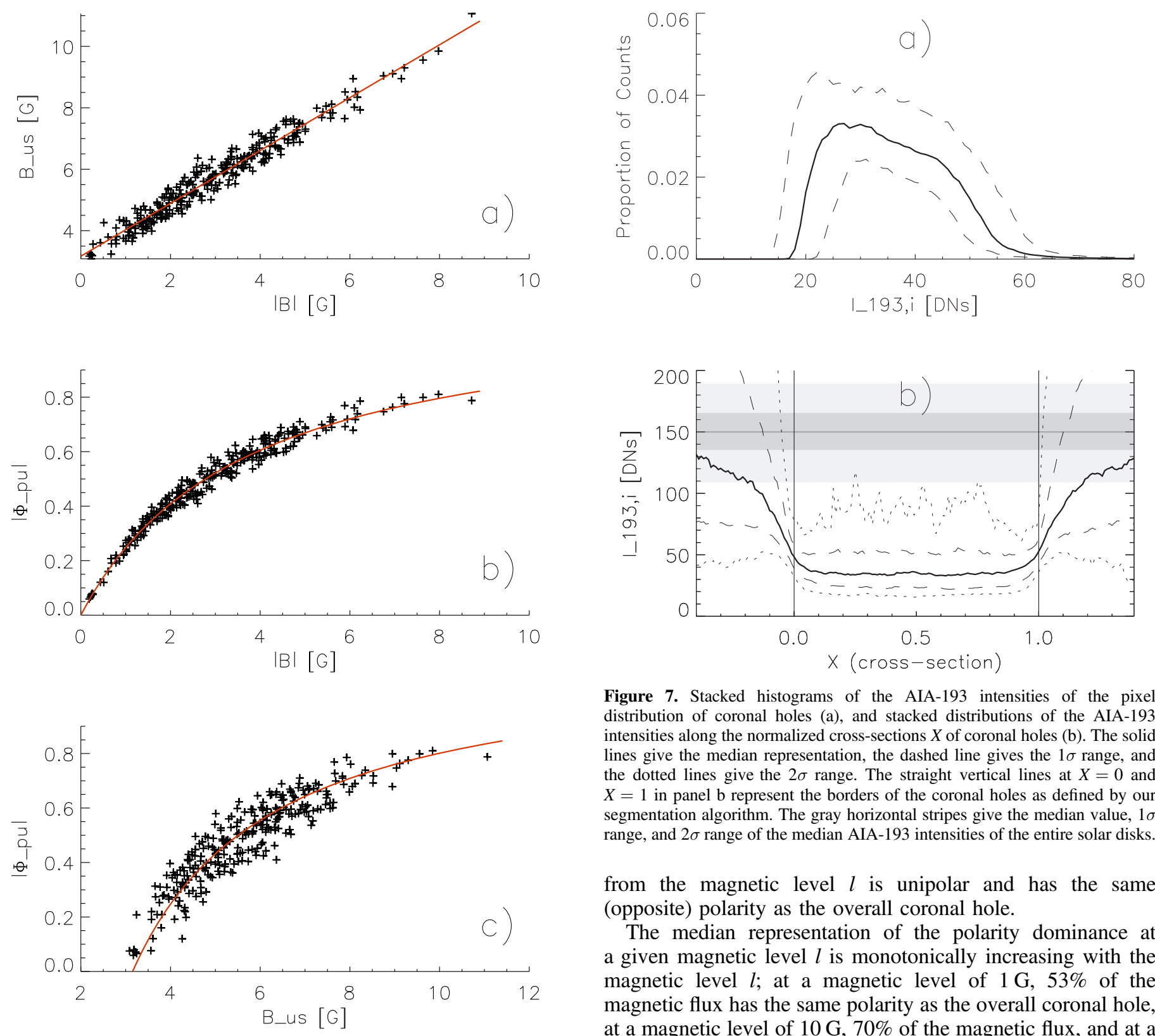

Figure 7. Stacked histograms of the AIA-193 intensities of the pixel distribution of coronal holes (a), and stacked distributions of the AIA-193 intensities along the normalized cross-sections $X$ of coronal holes (b). The solid lines give the median representation, the dashed line gives the $1 \sigma$ range, and the dotted lines give the $2 \sigma$ range. The straight vertical lines at $X=0$ and $X=1$ in panel $\mathrm{b}$ represent the borders of the coronal holes as defined by our segmentation algorithm. The gray horizontal stripes give the median value, $1 \sigma$ range, and $2 \sigma$ range of the median AIA-193 intensities of the entire solar disks.

from the magnetic level $l$ is unipolar and has the same (opposite) polarity as the overall coronal hole.

The median representation of the polarity dominance at a given magnetic level $l$ is monotonically increasing with the magnetic level $l$; at a magnetic level of $1 \mathrm{G}, 53 \%$ of the magnetic flux has the same polarity as the overall coronal hole, at a magnetic level of $10 \mathrm{G}, 70 \%$ of the magnetic flux, and at a magnetic level of $30 \mathrm{G}, 92 \%$ of the magnetic flux. The $2 \sigma$ range shows that even at low magnetic field density levels the polarity dominance is usually $>0.5$. This indicates that all magnetic levels of magnetic field density predominantly have the same polarity as the overall coronal hole. The magnetic flux arising from pixels with a weak magnetic field density is only slightly unbalanced toward the dominant polarity of the coronal hole, whereas the imbalance strongly increases with increasing magnetic field density, reaching a value of $\Phi_{\mathrm{pd}}(30)=0.92$ at a magnetic field density level of $30 \mathrm{G}$.

\subsection{Polarity Dominance at Given Magnetic Levels}

Figure 8 (b) shows the stacked polarity dominance at given magnetic field density levels $l, \Phi_{\mathrm{pd}}(l)$ for all coronal holes. The polarity dominance gives the fraction of the magnetic flux arising from pixels with magnetic field density $l$, which has the same polarity as the overall coronal hole. Therefore, a value of $\Phi_{\mathrm{pd}}(l)=0.5$ means that the magnetic flux arising from pixels with a magnetic field density $l$ is balanced; a value of $\Phi_{\mathrm{pd}}(l)=1\left(\Phi_{\mathrm{pd}}(l)=0\right)$ means that the magnetic flux arising

\subsection{Origin of the Unbalanced Magnetic Flux}

Figure 9(a) shows the stacked cumulative histograms of the absolute values of the magnetic field density $\left|B_{+}\right|$of coronal holes. In the median representation, $90 \%$ of all pixels within a coronal hole have an absolute magnetic field density $\left|B_{+}\right|<11 \mathrm{G}, 9 \%$ have an absolute magnetic field density $\left|B_{+}\right|$of $11-56 \mathrm{G}$, and $1 \%$ have an absolute magnetic field density $\left|B_{+}\right|>56 \mathrm{G}$. 

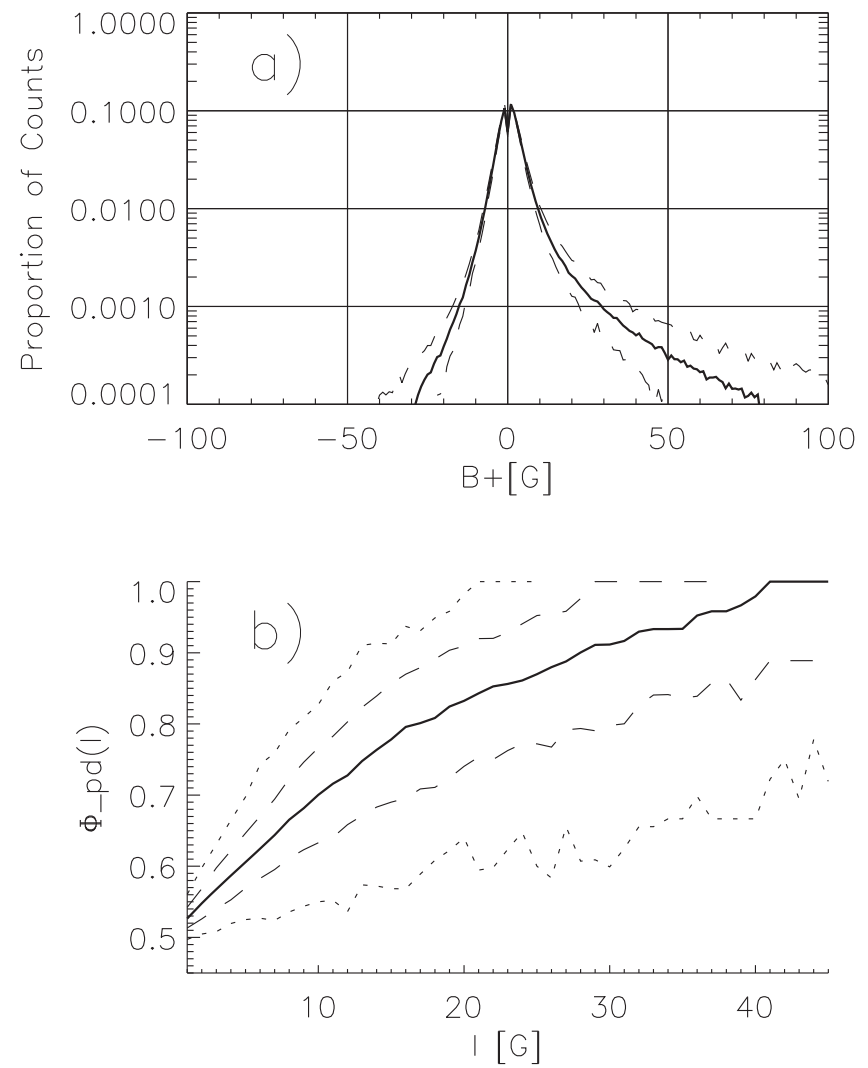

Figure 8. Stacked normalized histograms of the magnetic field densities $B_{+}$of coronal holes (a). Stacked polarity dominance $\Phi_{\mathrm{pd}}(l)$ of a given magnetic field density level $l$ vs. $l$ of coronal holes (b). Positive values of $B_{+}$belong to the dominant polarity of the corresponding coronal hole. The solid lines give the median values of all objects, the dashed lines the $1 \sigma$ range, and the dotted lines the $2 \sigma$ range.

Figure 9(b) shows the stacked plots of the fraction of the unbalanced magnetic flux $\Phi_{\mathrm{fu}}(b)$, which arises from pixels above a given threshold magnetic field density $b$ versus $b$. In the median representation, $81 \%$ of the unbalanced magnetic flux arises from pixels with an absolute magnetic field density $\left|B_{+}\right|>10 \mathrm{G}, 54 \%$ from pixels with $\left|B_{+}\right|>30 \mathrm{G}$, and $38 \%$ from pixels with $\left|B_{+}\right|>50 \mathrm{G}$. Therefore, most of the unbalanced magnetic flux of coronal holes arises from a small percentage of its area. This aspect is studied in further detail in the following section.

\section{Magnetic Flux Tubes}

In Figure 10, we show two samples, a small and a large sample coronal hole: the AIA-193 $\AA$ emission together with the coronal hole boundary determined (lower panel) and the corresponding line-of-sight magnetic field map from HMI (upper panel). We find that the pixels with a high magnetic field density, i.e., the regions where most of the magnetic flux comes from, are clustered. We interpret these clusters as magnetic flux tubes.

In this section, we analyze the distribution of the magnetic flux and areas of all flux tubes in the coronal holes under study. We count the numbers of flux tubes per area per coronal hole, calculate their summed unbalanced magnetic flux and area per coronal hole, and estimate the percentage of their summed unsigned magnetic flux, which is unbalanced.

Note that the flux tubes were extracted by a thresholding technique applied to the magnetograms; therefore, the
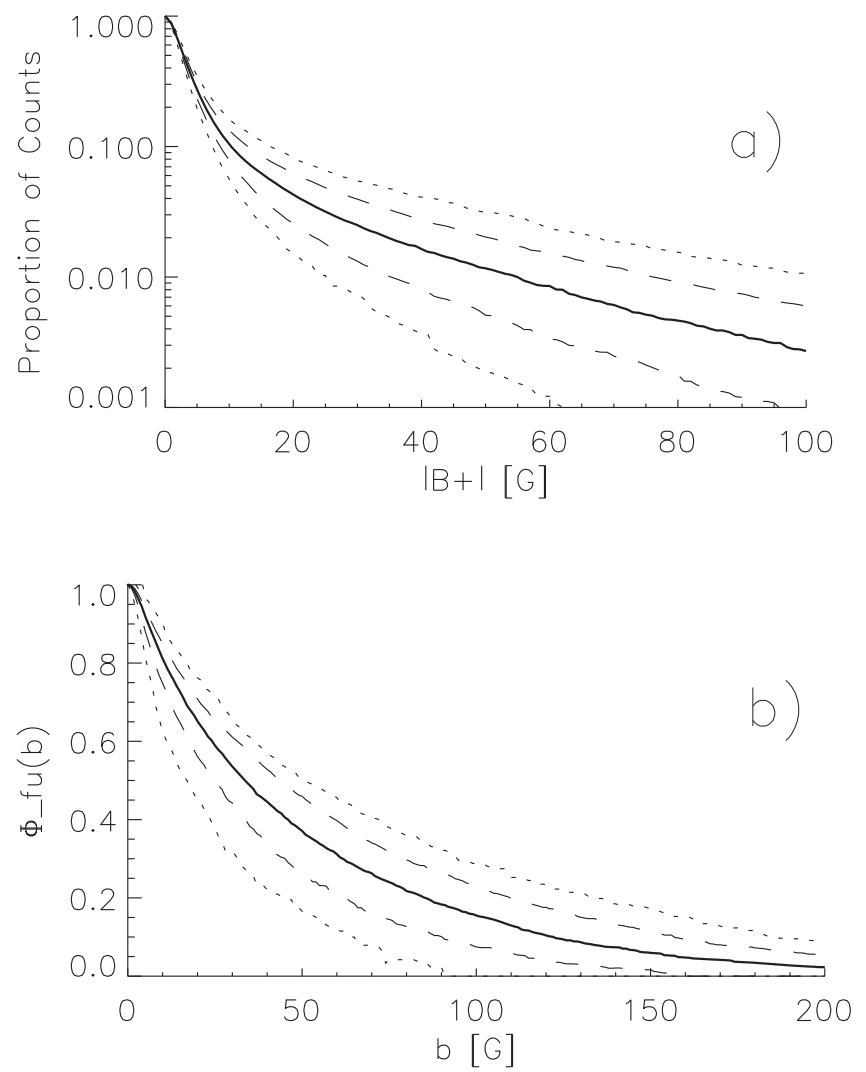

Figure 9. Stacked normalized cumulative histograms of the absolute magnetic field densities $\left|B_{+}\right|$of coronal holes (a). Stacked plots of the fraction of the unbalanced magnetic flux $\Phi_{\mathrm{fu}}(b)$ arising from pixels with $\left|B_{+}\right|>b$ on the overall unbalanced magnetic flux vs. $b$ of coronal holes (b). The solid line gives the median values of all objects, the dashed lines give the $1 \sigma$ range, and the dotted lines give the $2 \sigma$ range.

quantitative parameters depend on the actual extraction threshold. However, the qualitative results were found to be valid for various thresholds ranging from 10 to $80 \mathrm{G}$. At thresholds $>20 \mathrm{G}$, all flux tubes extracted in the coronal holes were unipolar, i.e., all pixels within the flux tube had the same polarity. Thus, the assumption of magnetic flux tubes is justified. In the following, we present the results for flux tubes extracted at thresholds, thr, of 10,30 , and $50 \mathrm{G}$.

\subsection{Magnetic Distribution of Flux Tubes}

In total, we extracted 26,409 flux tubes at $10 \mathrm{G}, 8850$ flux tubes at $30 \mathrm{G}$, and 4941 flux tubes at $50 \mathrm{G}$.

Figure 11(a) shows the histogram of the mean magnetic field densities $\bar{B}_{\text {o,thr }}$ of all flux tubes. Positive values correspond to flux tubes that have the same polarity as the dominant polarity of its coronal hole. At thresholds of $10 \mathrm{G}$, the mean magnetic field density is $18 \pm 20 \mathrm{G}$, at thresholds of $30 \mathrm{G} 58 \pm 29 \mathrm{G}$, and at thresholds of $50 \mathrm{G} 91 \pm 27 \mathrm{G}$. The positive values of the mean magnetic field densities, especially at the higher extraction thresholds, show that most flux tubes have the same dominant polarity as their coronal hole. The lower values of mean magnetic field densities at lower extraction thresholds arises from the higher number of weak magnetic flux tubes and the larger area per flux tube.

Figure 11(b) shows the distribution of the magnetic flux $\Phi_{o, \text { thr }}$ of all flux tubes. Most of the flux tubes have a small absolute magnetic flux: $75 \%$ have $\left|\Phi_{\circ, 10}\right|<1.0 \cdot 10^{19} \mathrm{Mx}$ at a 

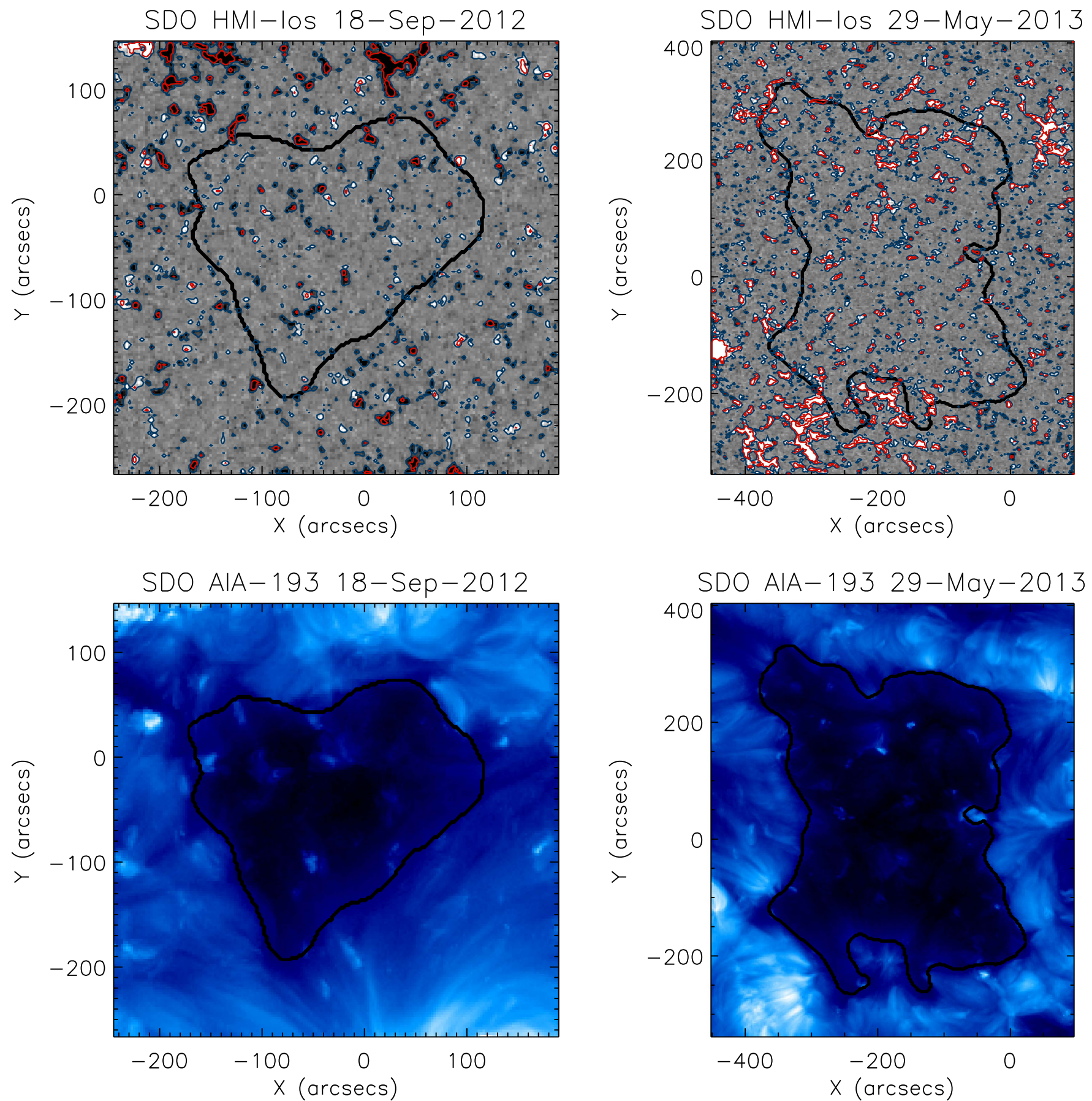

Figure 10. Magnetogram (scaled to $\pm 30 \mathrm{G}$ ) and $193 \AA$ A filtergram of a small sample coronal hole recorded on 2012 September 18 (left) and of a large sample coronal hole recorded on 2013 May 29 (right). The coronal hole boundaries are outlined. In the magnetogram, regions with an absolute magnetic field density of more than $10 \mathrm{G}$ are outlined in blue and of more than $50 \mathrm{G}$ in red.

flux-tube threshold of $10 \mathrm{G}, 75 \%$ have $\left|\Phi_{\circ, 30}\right|<2.2 \cdot 10^{19} \mathrm{Mx}$ at a threshold of $30 \mathrm{G}$, and $75 \%$ have $\left|\Phi_{\circ, 50}\right|<2.6 \cdot 10^{19} \mathrm{Mx}$ at a threshold of $50 \mathrm{G}$. However, there is also a small number of flux tubes with a large magnetic flux, with values as high as $5.3 \cdot 10^{20} \mathrm{Mx}$ at a threshold of $10 \mathrm{G}, 2.6 \cdot 10^{20} \mathrm{Mx}$ at a threshold of $30 \mathrm{G}$, and $1.6 \cdot 10^{20} \mathrm{Mx}$ at a threshold of $50 \mathrm{G}$.

\subsection{Relationship between Area and Magnetic Field Density of Flux Tubes}

Figure 11(c) shows the absolute mean magnetic field densities $\left|\bar{B}_{\circ, 50}\right|$ of all flux tubes extracted at $50 \mathrm{G}$ versus the flux-tube areas $A_{\circ, 50} .55 \%$ of all flux tubes have a small area of $A_{\circ, 50}<2.5 \cdot 10^{7} \mathrm{~km}^{2}$ and a small mean magnetic field density of $\left|\bar{B}_{\circ, 50}\right|<100 \mathrm{G}$. Flux tubes with a large area always have a medium to high mean magnetic field density. However, flux tubes with a very high mean magnetic field density usually have a small to medium area.

We further investigated the relationship between the absolute mean magnetic field density of flux tubes and their area extracted at $50 \mathrm{G}$ for each coronal hole individually. Figure 11(d) shows the scatter plot of the absolute mean magnetic field densities $\left|\bar{B}_{\circ, 50}\right|$ of flux tubes of a typical 

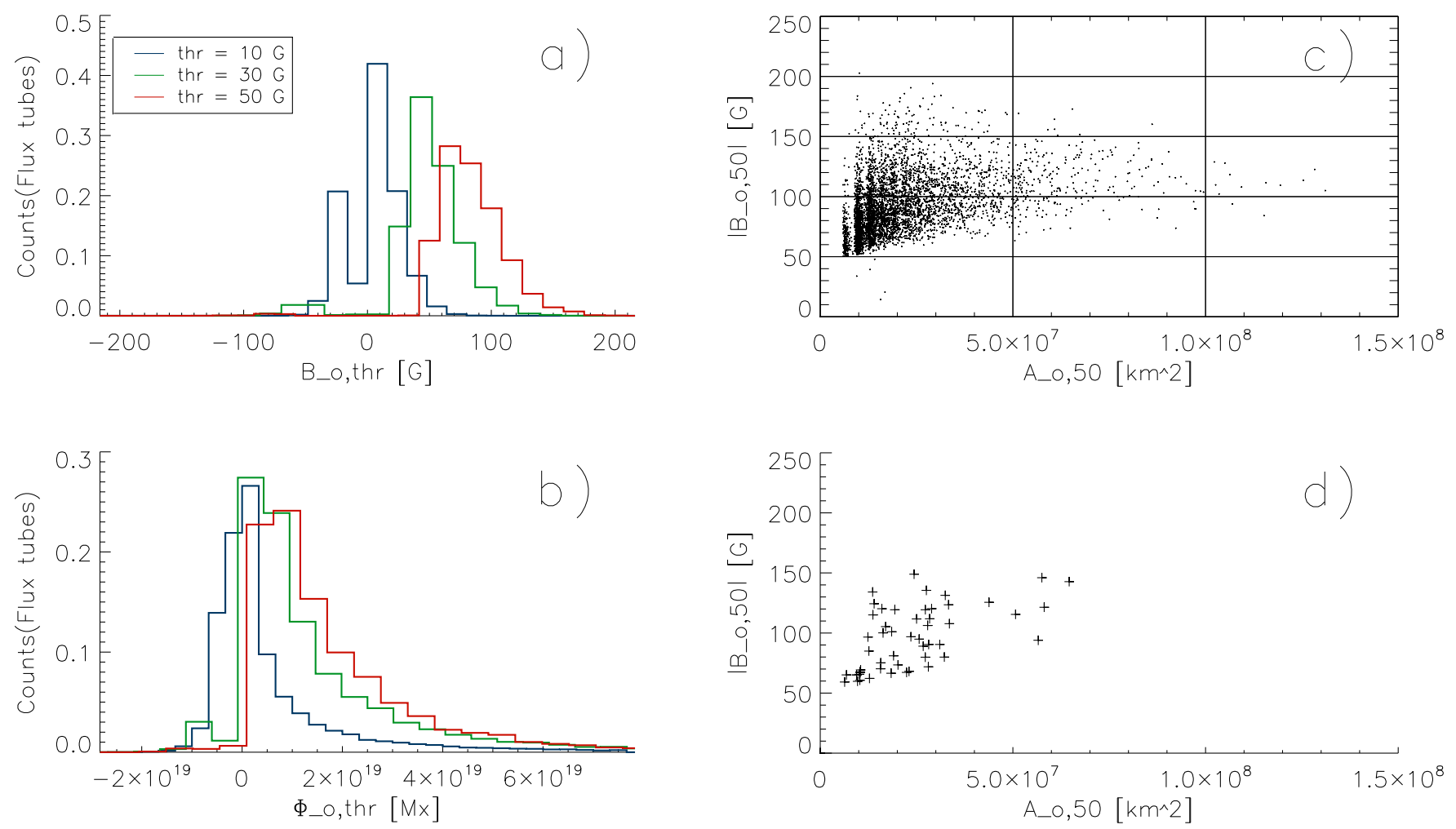

Figure 11. Distribution of the mean magnetic field densities (a) and magnetic flux (b), derived from all flux tubes extracted at $10 \mathrm{G}$ (blue), $30 \mathrm{G}$ (green), and $50 \mathrm{G}$ (red). Positive values belong to the dominant polarity of their source coronal hole. Scatter plot of the mean magnetic field density of all flux tubes vs. their areas derived from all flux tubes extracted at $50 \mathrm{G}$ (c), and derived for a single medium sized coronal hole (apparent on 2013/11/09; d).

medium sized coronal hole versus their areas $A_{\circ, 50}$. At medium size and large size coronal holes, a wide-spread relationship between the absolute mean magnetic field densities and the areas is often visible, with larger areas at higher mean magnetic field densities. This relationship can be explained by magnetic pressure: higher mean magnetic field densities are related to higher magnetic pressures within the flux tubes, and thus to statistically larger cross-sections. At small coronal holes, there are usually not enough flux tubes to make a meaningful statement on the correlation.

\subsection{Number of Flux Tubes per Coronal Hole}

Figure 12(a) shows the stacked plots of the number of flux tubes per coronal hole area, $\tilde{N}_{\circ, \text { thr }} / A$, versus the extraction threshold thr. Coronal holes have, on average, $28 \pm 3$ flux tubes extracted at $10 \mathrm{G}$ per $10^{10} \mathrm{~km}^{2}, 9 \pm 4$ flux tubes extracted at $30 \mathrm{G}$ per $10^{10} \mathrm{~km}^{2}$, and $5 \pm 3$ flux tubes extracted at $50 \mathrm{G}$ per $10^{10} \mathrm{~km}^{2}$.

\subsection{Flux Tubes as the Origin of Unbalanced Magnetic Flux}

In this section, we presume that magnetic flux tubes with the same polarity as the dominant polarity of the coronal hole do not close with quiet regions inside of the coronal hole, but only with flux tubes of opposite polarity inside of the coronal hole. This assumption is supported by the fact that coronal holes usually have the same dominant polarity at all magnetic scales (see Figure 8(b)), and that, therefore, the quiet coronal hole regions, on average, also have the same dominant polarity as the flux tubes with dominant polarity. In this case, the percentaged unbalanced magnetic flux of the flux tubes $\Phi_{\mathrm{pu}, \mathrm{thr}}$ gives a lower estimate on the fraction of the magnetic flux arising from flux tubes, which is unbalanced within the coronal hole boundaries.

Figure 12(b) shows the stacked plots of the percentaged unbalanced magnetic flux $\tilde{\Phi}_{\mathrm{pu} \text {,thr }}$ of the flux tubes per coronal hole versus the extraction threshold. The median line shows that for $50 \%$ of all coronal holes at least $85 \%$ of the magnetic flux arising from flux tubes is unbalanced at an extraction threshold of $10 \mathrm{G}$, at least $98 \%$ is unbalanced at an extraction threshold of $30 \mathrm{G}$, and the complete magnetic flux is unbalanced at an extraction threshold of $50 \mathrm{G}$. The bottom $1 \sigma$ line shows that for $82 \%$ of all coronal holes, at least $72 \%$ of the magnetic flux arising from flux tubes is unbalanced at an extraction threshold of $10 \mathrm{G}$, at least $93 \%$ at $30 \mathrm{G}$, and all the complete magnetic flux is unbalanced at $50 \mathrm{G}$. This means that, in general, most of the magnetic flux tubes in coronal holes, and, in particular, the strong magnetic flux tubes, are open. ${ }^{5}$

Note that Figure 9 can also be interpreted in the context of flux tubes. Figure 9(a) is proportional to the fraction of the coronal hole area that is covered by flux tubes extracted at a threshold thr $=b, \tilde{A}_{\circ \text {,thr }} / A$. Figure 9(b) then shows the fraction of magnetic flux to the unbalanced magnetic flux that arises from flux tubes, $\tilde{\Phi}_{o, \text { thr }} / \Phi$. The flux tubes extracted at thresholds of $10 \mathrm{G}$ cover $10 \%$ of the area of coronal holes, and carry $81 \%$ of the unbalanced magnetic flux. If we rise the threshold to $30 \mathrm{G}$, the flux tubes cover $2.5 \%$ of the area of coronal holes, and carry $54 \%$ of the unbalanced magnetic flux. At extraction thresholds of $50 \mathrm{G}$, the flux tubes cover $1.2 \%$ of the area of coronal holes, and carry $38 \%$ of the unbalanced magnetic flux.

\footnotetext{
5 However, the bottom $2 \sigma$ line shows that there is a small percentage of coronal holes with a small percentaged unbalanced magnetic flux of flux tubes, i.e., where a significant fraction of the flux tubes are closed.
} 

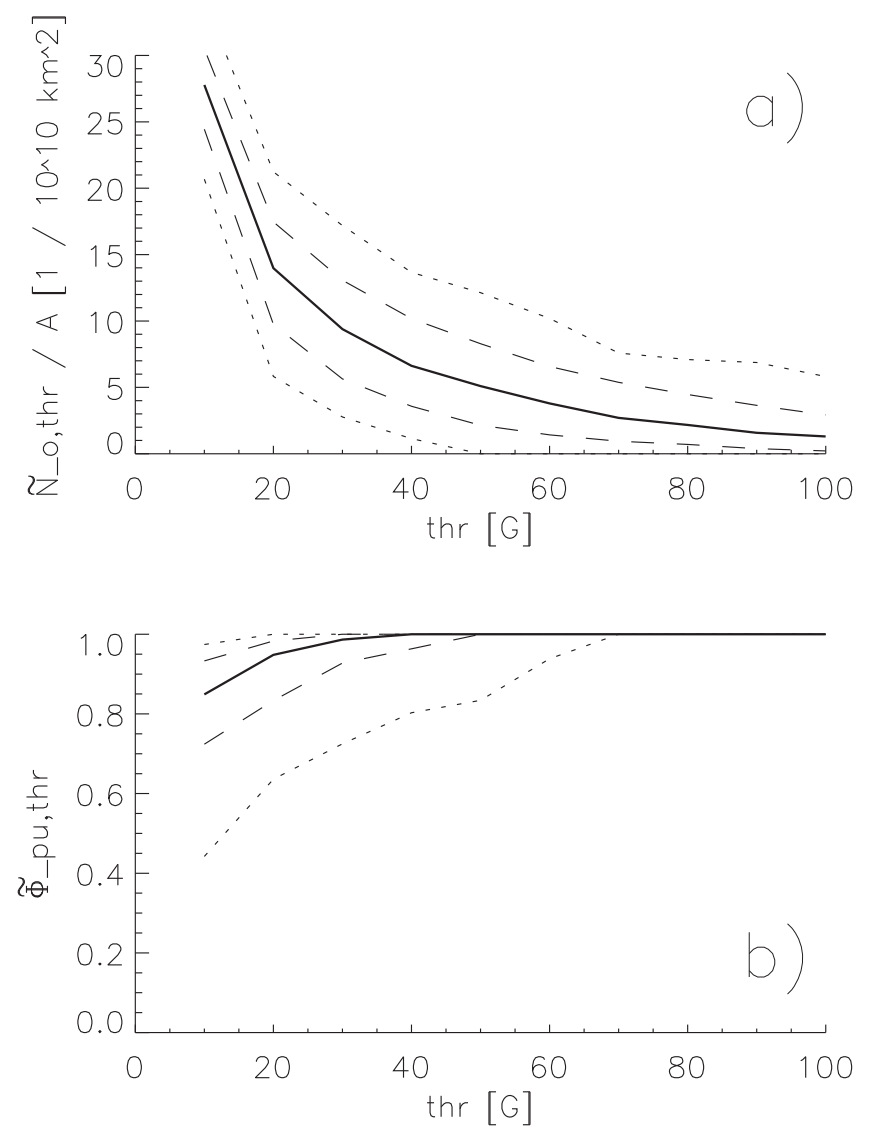

Figure 12. Stacked plots of the number of flux tubes extracted at thresholds, thr, per area vs. the extraction threshold, thr (a). Stacked plots of the percentaged unbalanced magnetic flux of all flux tubes per coronal hole extracted at thresholds, thr, vs. the extraction threshold, thr (b). The solid line gives the median values of all objects, the dashed lines the $1 \sigma$ range, and the dotted lines the $2 \sigma$ range.

This means that most of the unbalanced magnetic flux of coronal holes arise from very localized regions, i.e., magnetic flux tubes, covering only a small fraction of the total coronal hole area.

\section{Relationship between the Properties of Low-latitude Coronal Holes and Their Averaged Flux-tube Parameters}

In this section, we relate the averaged parameters of magnetic flux tubes per coronal hole (see Section 6) to the properties of the coronal holes (see Section 4). Here we only consider flux tubes extracted at a high threshold of $50 \mathrm{G}$ (Figures 13-15: left panels) and a low threshold of $10 \mathrm{G}$ (Figures 13-15: right panels). The results are qualitatively also valid for other extraction thresholds, whereby at lower extraction thresholds the correlation coefficients get higher and the scatter in the plots gets lower.

\subsection{Number of Flux Tubes}

Figure 13(a) shows the number of flux tubes extracted at $50 \mathrm{G} \tilde{N}_{\circ, 50}$ per coronal hole versus the area $A$ of coronal holes. The number of flux tubes depends linearly, but with a high scatter, on the area. Figure 13(b) shows the number of flux tubes per coronal hole normalized on the area of the coronal hole $\tilde{N}_{\circ, 50} / A$ versus the absolute values of the mean magnetic field density $|\bar{B}|$ of the coronal hole. The number of flux tubes per area depends linearly on the mean magnetic field density, with about 1.5 flux tubes per $10^{10} \mathrm{~km}^{2}$ at $|\bar{B}|=1 \mathrm{G}$ and 9 flux tubes per $10^{10} \mathrm{~km}^{2}$ at $|\bar{B}|=5 \mathrm{G}$. The total relationship can be expressed as

$$
N_{\circ, 50}=(-0.12+1.79|\bar{B}|[G]) A\left[10^{10} \mathrm{~km}^{2}\right],
$$

with an RMSE of 2.92 flux tubes.

In contrast, the number of flux tubes extracted at $10 \mathrm{G} \tilde{N}_{\circ, 10}$ depends mainly on the coronal hole area $A$ (Figure 13(c)), and only slightly on the mean magnetic field density $B$ of the coronal hole (Figure 13(d)), with

$$
N_{\circ, 10}=(25+0.77|\bar{B}|[G]) A\left[10^{10} \mathrm{~km}^{2}\right] .
$$

The RMSE is 10.5 flux tubes.

Altogether, the general number of flux tubes, i.e., extracted at a low extraction threshold of $10 \mathrm{G}$, depends mainly on the area of the coronal hole, whereas the number of strong magnetic flux tubes, i.e., extracted at $50 \mathrm{G}$, is also related to the mean magnetic field density of the coronal hole.

\subsection{Magnetic Field Densities}

Figure 14(a) shows the mean magnetic field density of the quiet coronal hole regions $\bar{B}_{\mathrm{qu}, 50}$, i.e., the coronal hole without the flux tubes extracted at $50 \mathrm{G}$, versus the averaged magnetic field density of the flux tubes extracted at $50 \mathrm{G} \tilde{B}_{\circ, 50}$. The mean magnetic field density of the quiet coronal hole correlates well with the averaged magnetic field density of the flux tubes, with a Spearman's rank correlation coefficient of 0.92 . However, this correlation could be artificially created by our flux-tube extraction algorithm in the case in which we only extracted the cores of the flux tubes and the outer layers of flux tubes are contained within our quiet coronal hole regions. Therefore, we cross-checked this relation at low extraction thresholds of $10 \mathrm{G}$ (Figure 14(d)) and found that the correlation still holds at a Spearman's rank correlation coefficient of 0.96 . This finding shows that strong flux tubes within the coronal hole result in a distinctly increased mean magnetic field density of the quiet coronal hole region. It also implies that the magnetic field of the flux tubes and of the quiet coronal hole area are coupled.

Figure 14(b) shows the averaged magnetic field density derived from all flux tubes extracted at $50 \mathrm{G}$ within a coronal hole $\tilde{B}_{\circ, 50}$ versus the mean magnetic field density of the coronal hole $\bar{B}$. The averaged magnetic field density of the flux tubes extracted at $50 \mathrm{G}$ correlates strongly with the mean magnetic field density of the overall coronal hole, with a Spearman's rank correlation coefficient of 0.93 . By decreasing the flux-tube extraction threshold to $10 \mathrm{G}$, the correlation coefficient even increases to 0.99 (Figure 14(e)). The relationships can be expressed as

$$
\begin{aligned}
\tilde{B}_{\circ, 50}[\mathrm{G}] & =\frac{115 \bar{B}[\mathrm{G}]}{0.7 \mathrm{G}+|\bar{B}|[\mathrm{G}]}, \\
\tilde{B}_{\circ, 10}[\mathrm{G}] & =\frac{58 \bar{B}[\mathrm{G}]}{3.9 \mathrm{G}+|\bar{B}|[\mathrm{G}]},
\end{aligned}
$$

with an RMSE of $10.7 \mathrm{G}$ and a mean absolute percentage error (MAPE) of $8.6 \%$ for flux tubes extracted at $50 \mathrm{G}$ and an RMSE of $1.6 \mathrm{G}$ and an MAPE of $6.8 \%$ for flux tubes extracted at $10 \mathrm{G}$. Note that these correlations result from the correlation between the average magnetic field density of flux tubes and the mean magnetic field density of the quiet coronal hole regions. 

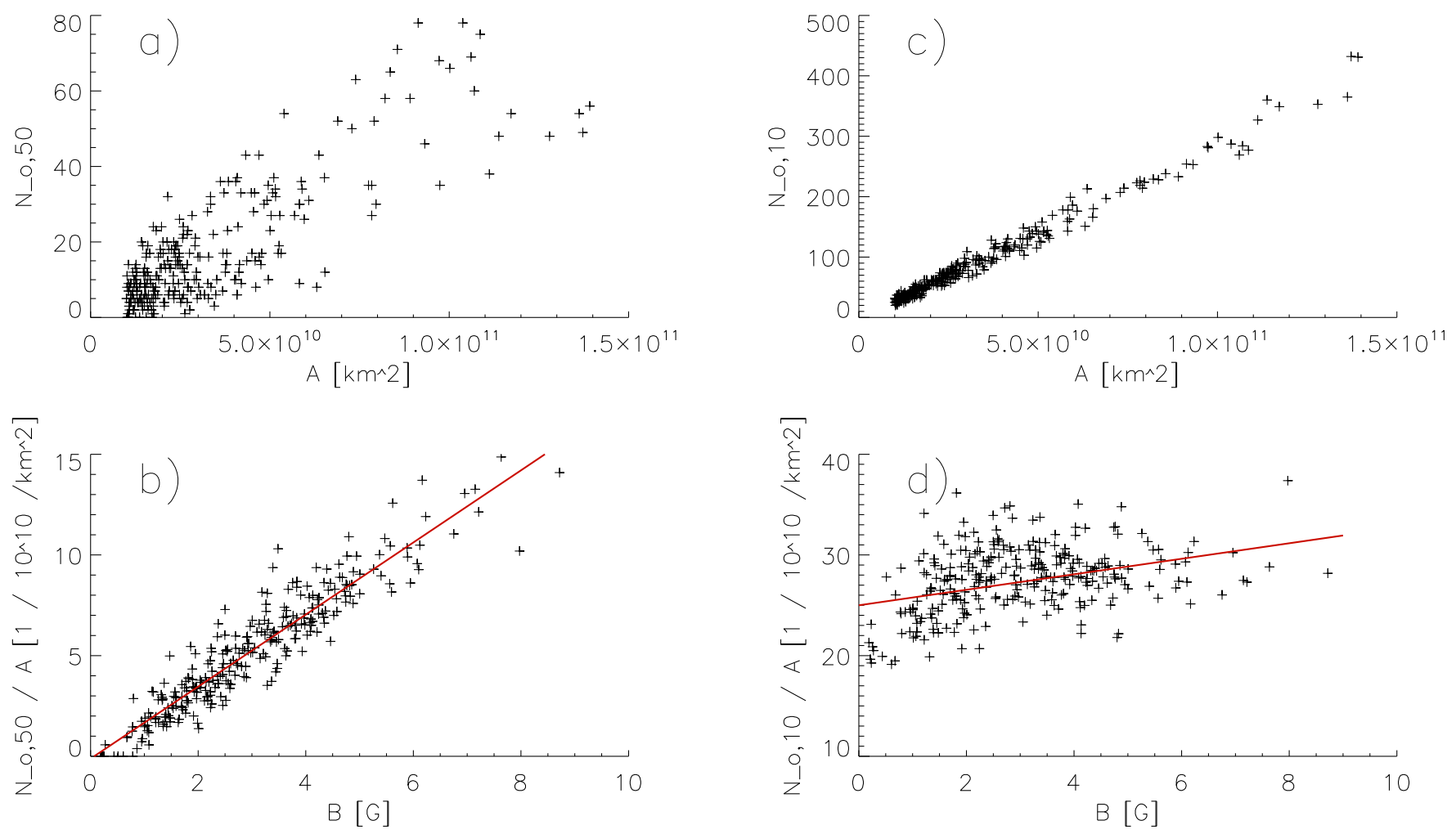

Figure 13. Scatter plot of the number of flux tubes per coronal hole vs. the area of the coronal hole (upper panel), and of the number of flux tubes per area vs. the absolute values of the mean magnetic magnetic field density of the coronal hole (lower panel), derived for flux tubes extracted at $50 \mathrm{G}$ (left panel) and $10 \mathrm{G}$ (right panel). In panel (b) and (d), the corresponding fits given by Equations (22) and (23) are over-plotted in red.

Figure 14(c) shows the mean area per flux tube extracted at $50 \mathrm{G}$ derived from all flux tubes within a coronal hole $\tilde{A}_{\circ, 50} / \tilde{N}_{\circ, 50}$ versus the mean magnetic field density of the coronal hole $|\bar{B}|$. The mean area per flux tube extracted at $50 \mathrm{G}$ correlates well with the mean magnetic field density of the coronal hole, with a Pearsons correlation coefficient of 0.64. By decreasing the flux-tube extraction threshold to $10 \mathrm{G}$, the correlation coefficient increases to 0.93 (Figure 14(f)). The relationships can be expressed as

$$
\begin{aligned}
& \frac{A_{\circ, 50}}{N_{\circ, 50}}\left[\mathrm{~km}^{2}\right]=1.28 \cdot 10^{7}+2.13 \cdot 10^{6}|\bar{B}|[\mathrm{G}], \\
& \frac{A_{\circ, 10}}{N_{\circ, 10}}\left[\mathrm{~km}^{2}\right]=1.58 \cdot 10^{7}+6.08 \cdot 10^{6}|\bar{B}|[\mathrm{G}],
\end{aligned}
$$

with an RMSE of $3.8 \cdot 10^{6} \mathrm{~km}^{2}$ and an MAPE of $15 \%$ for flux tubes extracted at $50 \mathrm{G}$ and an RMSE of $3.6 \cdot 10^{6} \mathrm{~km}^{2}$ and an MAPE of $8 \%$ for flux tubes extracted at $10 \mathrm{G}$. These relationships can simply be explained by magnetic pressure: a high mean magnetic field density of the coronal hole is related to high magnetic field densities in the flux tubes (see Figures 14(b) and (e)). A high mean magnetic field density of flux tubes results in a high magnetic pressure within the flux tube, and therefore in a large cross-section of the flux tube under the condition of comparably constant total pressure of the surrounding of the flux tube.

Altogether, the mean area per flux tube correlates well with the mean magnetic field density of the coronal hole. Furthermore, the mean magnetic field density of the coronal hole, the mean magnetic field density derived from the quiet coronal hole regions, and the averaged magnetic field density derived from all flux tubes within a coronal hole strongly correlate with each other. At lower extraction thresholds, i.e., by taking weaker flux tubes into account, the goodness of the relationships even enhance: the correlation coefficients increase and the MAPE decreases.

\subsection{Unbalanced Magnetic Flux}

Figure 15(a) shows the unbalanced magnetic flux derived from all flux tubes extracted at $50 \mathrm{G}$ within a coronal hole $\tilde{\Phi}_{0,50}$ versus the unbalanced magnetic flux $\Phi$ of the coronal hole. The unbalanced magnetic flux of the flux tubes correlates with the unbalanced magnetic flux of the complete coronal hole, with a Spearmans rank correlation coefficient of 0.99. By decreasing the flux-tube extraction threshold to $10 \mathrm{G}$, the Spearmans rank correlation coefficient stays at 0.99 . The relationships can be expressed as

$$
\begin{aligned}
& \tilde{\Phi}_{\circ, 50}[\mathrm{Mx}]=0.42 \Phi[\mathrm{Mx}], \\
& \tilde{\Phi}_{\mathrm{o}, 10}[\mathrm{Mx}]=0.84 \Phi[\mathrm{Mx}],
\end{aligned}
$$

with an RMSE of $8.9 \cdot 10^{19} \mathrm{Mx}$ and an MAPE of $79 \%$ for flux tubes extracted at $50 \mathrm{G}$, and an RMSE of $4.7 \cdot 10^{19} \mathrm{Mx}$ and an MAPE of $7 \%$ for flux tubes extracted at $10 \mathrm{G}$. This means that about $42 \%(84 \%)$ of the total unbalanced magnetic flux of coronal holes arises from flux tubes extracted at $50 \mathrm{G}(10 \mathrm{G})$. Note that these values slightly differ from the values derived in Section 6.4 (38\% for flux tubes extracted at $50 \mathrm{G}$, and $81 \%$ for flux tubes extracted at $10 \mathrm{G}$ ), since the least-square fit, which was used to derive Equations (28) and (29), privileges coronal holes with high magnetic fluxes. The high MAPE of Equation (28) arises from the large amount of small coronal 

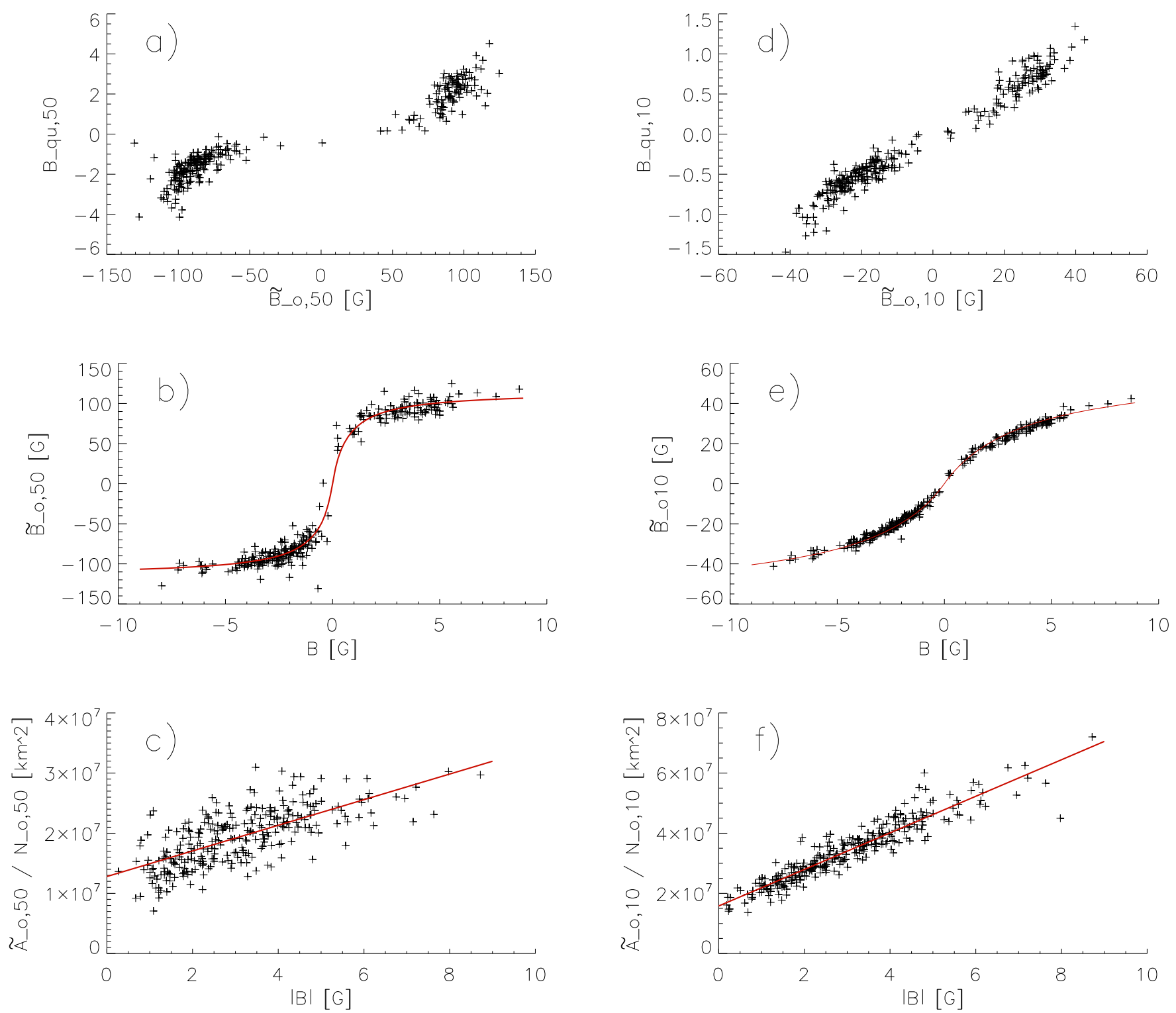

Figure 14. Scatter plot of the averaged magnetic field density of all flux tubes per coronal hole vs. the mean magnetic field density of the coronal hole quiet regions (upper panel), of the averaged mean magnetic field density of all flux tubes per coronal hole vs. the mean magnetic field density of the coronal hole (middle panel), and of the mean area per flux tube vs. the mean magnetic field density of coronal holes (lower panel), derived for flux tubes extracted at $50 \mathrm{G}$ (left panel) and $10 \mathrm{G}$ (right panel). In panel (b), (c), (e), and (f), the corresponding fits given by Equations (24)-(27) are over-plotted in red.

holes in our data set, which have small magnetic fluxes and are therefore not described well by Equation (28). The MAPE of Equation (29) is significantly decreased in comparison to Equation (28), since the flux tubes extracted at $10 \mathrm{G}$ already hold most of the unbalanced magnetic flux of the overall coronal hole.

Figure 15(a) shows the contribution of the flux tubes extracted at $50 \mathrm{G}$ on the unbalanced magnetic flux of the coronal hole, $\tilde{\Phi}_{\circ, 50} / \Phi$, versus the absolute value of the average magnetic field density of the flux tubes $\left|\tilde{B}_{\circ, 50}\right|$. The proportion of the magnetic flux arising from the flux tubes on the unbalanced magnetic flux of the coronal hole depends on the averaged magnetic field density of the flux tubes: at a high averaged magnetic field density of all flux tubes within a coronal hole about $60 \%$ of the unbalanced magnetic flux arises from flux tubes, at a low averaged magnetic field density only about to 20\%; the Spearman's rank correlation coefficient is
0.63. By decreasing the flux-tube extraction threshold to $10 \mathrm{G}$, the amount of unbalanced magnetic flux arising from the flux tubes increases to about $70 \%-87 \%$ dependent on the averaged magnetic field density of the flux tubes; the Spearman's rank correlation coefficient is 0.62 . Taking these correlation into account, Equations (28) and (29) improve to

$$
\begin{gathered}
\tilde{\Phi}_{\circ, 50}[\mathrm{Mx}]=\left(-0.12+0.0055\left|\tilde{B}_{\circ, 50}\right|[\mathrm{G}]\right) \Phi[\mathrm{Mx}], \\
\tilde{\Phi}_{\circ, 10}[\mathrm{Mx}]=\left(0.72+0.0041\left|\tilde{B}_{\circ, 10}\right|[\mathrm{G}]\right) \Phi[\mathrm{Mx}],
\end{gathered}
$$

with an RMSE of $8.4 \cdot 10^{19} \mathrm{Mx}$ and an MAPE of $30 \%$ for flux tubes extracted at $50 \mathrm{G}$, and an RMSE of $3.2 \cdot 10^{19} \mathrm{Mx}$ and an MAPE of $4.5 \%$ for flux tubes extracted at $10 \mathrm{G}$.

In summary, all the relationships in this section show that the magnetic characteristics of coronal holes are set by its magnetic flux tubes. A high mean magnetic field density of flux tubes results in a large mean area per flux tube, and thus to a high magnetic flux per flux tube and to high amount of 

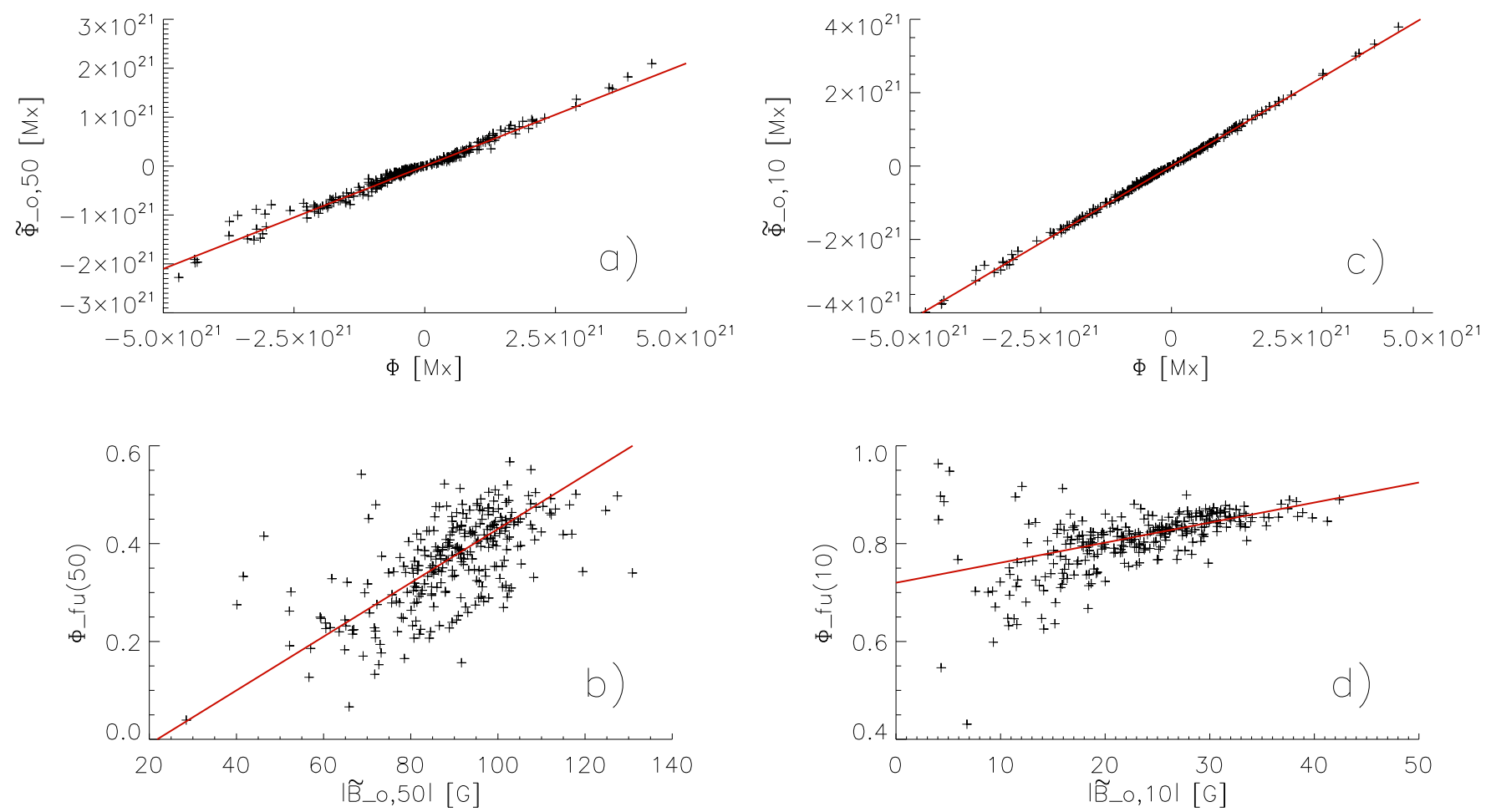

Figure 15. Scatter plot of the summed unbalanced magnetic flux of all flux tubes per coronal hole vs. the unbalanced magnetic flux of the coronal hole (upper panel), and of the relative amount of unbalanced magnetic flux arising from flux tubes on the unbalanced magnetic flux of coronal holes vs. the averaged mean magnetic field density of flux tubes per coronal hole (lower panel), derived for flux tubes extracted at $50 \mathrm{G}$ (left panel) and $10 \mathrm{G}$ (right panel). The corresponding fits given by Equations (28)-(31) are over-plotted in red.

unbalanced magnetic flux arising from flux tubes. In addition, a high magnetic field density of flux tubes is related to a high mean magnetic field density of the quiet coronal hole regions, and therefore to high mean magnetic field density of the overall coronal hole.

\section{Summary and Discussion}

We studied the statistics of characteristic properties of 288 records of low-latitude coronal holes observed during the period of 2011 January 01-2013 December 31, the EUV intensity and magnetic field distribution within the coronal holes, and the distribution of magnetic flux tubes. The main findings are as follows.

1. The mean magnetic field density of coronal holes ranged from 0.2 to $8.7 \mathrm{G}$ with a mean value of $3.0 \pm 1.6 \mathrm{G}$, the percentaged unbalanced magnetic flux from $6 \%$ to $81 \%$ with a mean value of $49 \pm 16 \%$.

2. The mean magnetic field density, the mean unsigned magnetic field density, and the percentaged unbalanced magnetic flux of coronal holes depend strongly on each other in pairs at correlation coefficients of $>0.92$ (see Figure 6).

3. Coronal holes usually have the same polarity on all magnetic levels: the polarity dominance at a given magnetic level $l, \Phi_{\mathrm{pd}}(l)$, is at all magnetic levels $l$ for almost all coronal holes always $>0.5$ (see Figure $8(b)$ ).

4. The magnetic flux of coronal holes is concentrated in magnetic flux tubes. The flux tubes extracted at $50 \mathrm{G}$ $(10 \mathrm{G})$ cover only $1 \%(10 \%)$ of the area of coronal holes, but contain $38 \%(81 \%)$ of the unbalanced magnetic flux of coronal holes (see Figure 9).
5. In most coronal holes, almost all magnetic flux tubes, and especially the strong magnetic flux tubes, do not close within the coronal hole boundaries (see Figure 12(b)).

6. The mean magnetic field densities of coronal holes depend strongly on the averaged magnetic field density derived from all their flux tubes within the coronal hole, and the unbalanced magnetic flux of coronal holes depends strongly on the total unbalanced magnetic flux derived from all flux tubes within the coronal hole, at correlation coefficients $>0.93$ (see Figures 14(b), (e), Figures 15(a), (c)).

The mean magnetic field densities derived in our data set are significantly smaller than the values derived by Harvey et al. (1982, 3-36 G) and Wang (2009, about 20 G) for solar maxima, and match better with the values they derived for solar minima (1-7 G, respectively about $5 \mathrm{G})$. Although the selection procedure of the data sets differ-we took all lowlatitude coronal holes with areas of $A>10^{10} \mathrm{~km}^{2}$, whereas Wang (2009) took all coronal holes on the solar disk, and Harvey et al. (1982) took 63 selected low-latitude coronal holes -it seems unlikely that this difference is only due to the different selection of the data sets. We think that the low mean magnetic field densities of our low-latitude coronal holes examined near solar maximum are related to the weak polar magnetic field strength in the solar minimum before solar cycle 24 and the very low solar activity in the actual solar cycle 24 (e.g., review by Basu 2013). However, instrumental effects of the different magnetographs used by the different authors also cannot be excluded.

The characteristic parameters of coronal holes, i.e., the areas, the mean AIA-193 intensities, and the magnetic parameters, could further be related to the age of coronal holes. Since the 
area and the mean magnetic field density of coronal holes depends on the age of coronal holes (Bohlin 1977; Bohlin \& Sheeley 1978), the unbalanced magnetic flux and the percentaged unbalanced magnetic flux also depend on the age of coronal holes. If we assume for simplicity that the entirety of small coronal holes belongs to the birth and end of coronal holes and the entirety of large coronal holes to the middle of their lifetime, the different range of scatter in the characteristic parameters of small to large coronal holes might simply be explained by the different phases in their lifetimes.

We found that the average parameters of magnetic flux tubes extracted at each extraction threshold, thr, are strongly correlated to the magnetic parameters of the coronal hole. This implies that the average parameters of the magnetic flux tubes extracted at different thresholds, thr, are strongly correlated, though the number of flux tubes and their amount on the unbalanced magnetic flux strongly changes with thr. In addition, a high average magnetic field density of flux tubes is always related to comparably high magnetic field densities of quiet coronal hole regions, i.e., the coronal hole region without the flux tubes. This relationship results in a distinct polarity at all magnetic levels within the coronal hole and to a more pronounced polarity and thus a higher percentaged unbalanced magnetic flux at higher mean magnetic field densities of the overall coronal hole. Thus, the magnetic characteristics of the flux tubes set the magnetic characteristics of the overall coronal hole.

The magnetic flux tubes we identified by a thresholding technique are likely to be related to the coronal magnetic funnels described in Wiegelmann et al. (2005) and Tu et al. (2005). The magnetic funnels are rooted near supergranulation boundaries in the chromospheric network lanes and are the origin of plasma outflows that form high-speed solar wind streams. The outflow velocity is statistically higher in regions with a higher magnetic field density in the underlying photosphere (Xia et al. 2004). If we assume that our magnetic flux tubes coincide with the foot points of the magnetic funnels, then we can speculate that the averaged plasma outflow velocity might be related to the averaged magnetic field density of the magnetic flux tubes, and therefore might also be related to the mean magnetic field density of the overall coronal hole.

Finally, we note that the very strong correlation between the averaged magnetic parameters of flux tubes extracted at various magnetic levels, the quiet coronal hole regions, and the overall coronal hole parameters $(c c>0.9)$ show that there has to be an underlying mechanism that sets the distribution of flux tubes and that relates the averaged magnetic field density of flux tubes to the mean magnetic field density of quiet coronal hole regions in an uniquely defined way. Furthermore, this mechanism has to contain the strong dependency between the mean magnetic field density, the mean unsigned magnetic field density, and the percentaged unbalanced magnetic flux of coronal holes $(c c>0.92)$. This controlling mechanism is yet not known; the understanding of this mechanism might greatly improve our knowledge of coronal holes and the associated high-speed solar wind streams.

The $S D O /$ AIA images and SDO/HMI images are available by courtesy of NASA/SDO and the AIA, EVE, and HMI science teams. A.V. acknowledges financial support by Austrian Science Fund (FWF): P24092-N16. B.V. received funding from the Croatian Science Foundation under project 6212 "Solar and Stellar Variability."

\section{References}

Altschuler, M. D., Trotter, D. E., \& Orrall, F. Q. 1972, SoPh, 26, 354 Basu, S. 2013, JPhCS, 440, 012001

Bohlin, J. D. 1977, SoPh, 51, 377

Bohlin, J. D., \& Sheeley, N. R., Jr. 1978, SoPh, 56, 125

Boucheron, L. E., Valluri, M., \& McAteer, R. T. J. 2016, SoPh, 291, 2353

Caplan, R. M., Downs, C., \& Linker, J. A. 2016, ApJ, 823, 53

Couvidat, S., Schou, J., Hoeksema, J. T., et al. 2016, SoPh, 291, 1887

Fainshtein, V. G., \& Kaigorodov, A. P. 1994, SoPh, 152, 429

Gnevyshev, M. N. 1963, SvA, 7, 311

Gnevyshev, M. N. 1977, SoPh, 51, 175

Harvey, K. L., Harvey, J. W., \& Sheeley, N. R., Jr. 1982, SoPh, 79, 149

Hassler, D. M., Dammasch, I. E., Lemaire, P., et al. 1999, Sci, 283, 810

Kohl, J. L., Noci, G., Cranmer, S. R., \& Raymond, J. C. 2006, A\&ARv, 13, 31 Kojima, M., Tokumaru, M., Fujiki, K., et al. 2007, in ASP Conf. Ser. 369, New

Solar Physics with Solar-B Mission, ed. K. Shibata, S. Nagata, \& T. Sakurai (San Francisco, CA: ASP), 549

Krista, L. D., \& Gallagher, P. T. 2009, SoPh, 256, 87

Lemen, J. R., Title, A. M., Akin, D. J., et al. 2012, SoPh, 275, 17

Levine, R. H., Altschuler, M. D., \& Harvey, J. W. 1977, JGR, 82, 1061

Levine, R. H., Altschuler, M. D., Harvey, J. W., \& Jackson, B. V. 1977, ApJ, 215,636

Lowder, C., Qiu, J., Leamon, R., \& Liu, Y. 2014, ApJ, 783, 142

Nolte, J. T., Krieger, A. S., Timothy, A. F., et al. 1976, SoPh, 46, 303

Pötzi, W., Veronig, A. M., Riegler, G., et al. 2015, SoPh, 290, 951

Reiss, M. A., Temmer, M., Veronig, A. M., et al. 2016, SpWea, 14, 495

Rotter, T., Veronig, A. M., Temmer, M., \& Vršnak, B. 2012, SoPh, 281, 793

Scherrer, P. H., Schou, J., Bush, R. I., et al. 2012, SoPh, 275, 207

Schou, J., Scherrer, P. H., Bush, R. I., et al. 2012, SoPh, 275, 229

Tu, C.-Y., Zhou, C., Marsch, E., et al. 2005, Sci, 308, 519

Verbeeck, C., Delouille, V., Mampaey, B., \& De Visscher, R. 2014, A\&A, 561, A29

Wang, Y.-M. 2009, SSRv, 144, 383

Wang, Y.-M., \& Sheeley, N. R., Jr. 1990, ApJ, 355, 726

Wiegelmann, T., \& Solanki, S. K. 2004, SoPh, 225, 227

Wiegelmann, T., Xia, L. D., \& Marsch, E. 2005, A\&A, 432, L1

Xia, L. D., Marsch, E., \& Wilhelm, K. 2004, A\&A, 424, 1025 